\title{
MODULUS OF SURFACE FAMILIES AND THE RADIAL STRETCH IN THE HEISENBERG GROUP
}

\author{
IOANNIS D. PLATIS
}

\begin{abstract}
We develop a modulus method for surface families inside a domain in the Heisenberg group and we prove that the stretch map between two Heisenberg spherical rings is a minimiser for the mean distortion among the class of contact quasiconformal maps between these rings which satisfy certain boundary conditions.
\end{abstract}

\section{Introduction and Statement of Results}

In the classical theory of quasiconformal mappings of the complex plane $\mathbb{C}$, various tools have been developed for the solution of the so called extremal problems: given a family $\mathcal{F}$ of quasiconformal mappings $f$ between two given regions $\Omega$ and $\Omega^{\prime}$ of $\mathbb{C}$ subject to boundary conditions, find a minimiser of the maximal distortion

$$
K_{f}=\sup _{p \in \Omega} K_{f}(p), \quad K_{f}(p)=\frac{\left|f_{z}(p)\right|+\left|f_{\bar{z}}(p)\right|}{\left|f_{z}(p)\right|-\left|f_{\bar{z}}(p)\right|}, \quad f \in \mathcal{F},
$$

or a minimiser of the mean distortion

$$
\mathfrak{M}\left(f, \rho_{0}\right)=\frac{\iint_{\Omega} K_{f}(p) \rho_{0}^{2}(p) d \mathcal{L}^{2}(p)}{\iint_{\Omega} \rho_{0}^{2}(p) d \mathcal{L}^{2}(p)}, \quad f \in \mathcal{F},
$$

where $\rho_{0}: \Omega \rightarrow \mathbb{R}^{+}$is a positive Borel function related to the geometry of $\Omega$. That is, in the first case, we wish to find an $f_{0} \in \mathcal{F}$ so that $K_{f_{0}} \leq K_{f}$ for every $f \in \mathcal{F}$, whereas in the second case our minimiser has to satisfy $\mathfrak{M}\left(f_{0}, \rho_{0}\right) \leq \mathfrak{M}\left(f, \rho_{0}\right)$ for every $f \in \mathcal{F}$. Motivation to solve problems like these comes from various sources, we mention for instance theory of elasticity. The study of the first problem goes back to Grötzsch, [8], Teichmüller, [21, Strebel, [19], to mention only a few. Modulus methods for curve families and quadratic differentials theory constitute classical tools for the solution of this problem. The second problem is more difficult; as it was shown in [14] there are examples where a minimiser for the mean distortion does not exist. Nevertheless, several other methods have been deployed for the study of this problem, see for instance [1, [3], 9], [14]. For an overview of the section of Teichmüller theory concerning extremal problems, in particular quadratic differentials theory, we refer the reader to the books of Strebel [20] and Gardiner and Lakic, [7]; for modulus methods we refer to the book of Vasil'ev, [22].

In contrast to the classical theory where a rather rich machinery is available for the solution of minimisation problems of the aforementioned nature, in the Heisenberg group (or, more generally, in the sub-Riemannian) case the picture is not analogous. However, the combination of the powerful Korányi-Reimann theory of quasiconformal mappings of the Heisenberg group with moduli methods seem to yield interesting results. In this paper is to propose a method of modulus of surface families inside the Heisenberg group in order to minimise a mean distortion functional; we describe this method below.

Key words and phrases. Heisenberg group, extremal quasiconformal mappings, mean distortion. 2010 Mathematics Subject Classification: 30L10, 30C75. 
For the moment, we recall in brief a few facts about the Heisenberg group $\mathfrak{H}$; for details, see Section 2, $\mathfrak{H}$ is $\mathbb{C} \times \mathbb{R}$ with group law

$$
(z, t) *(w, s)=(z+w, t+s+2 \Im(\bar{w} z))
$$

and it admits a natural left invariant metric (the Heisenberg distance) defined by

$$
d_{\mathfrak{H}}(p, q)=\left\|p^{-1} * q\right\|, \quad p=(z, t), q=(w, s) \in \mathfrak{H},
$$

where $\|(z, t)\|=\left.|| z\right|^{2}-\left.i t\right|^{1 / 2}$. The general metric definition of a quasiconformal mapping due to Mostow, [15], applies for the Heisenberg group $\mathfrak{H}$ when it is considered as a metric space with metric $d_{\mathfrak{H}}$.

Definition 1.1. (Metric definition) A homeomorphism $f: \Omega \rightarrow \Omega^{\prime}$ between domains $\Omega$ and $\Omega^{\prime}$ in $\mathfrak{H}$ is quasiconformal if

$$
\operatorname{ess} \sup _{p \in \Omega} H(p)<\infty,
$$

where

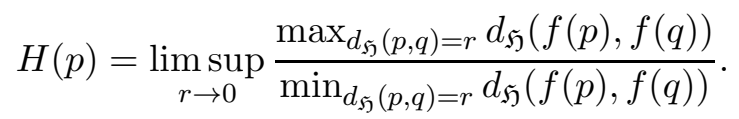

It is called $K$-quasiconformal if there is a constant $K \geq 1$ such that

$$
\text { ess } \sup _{p \in \Omega} H(p) \leq K \text {. }
$$

In analogy to the complex case, there are equivalent analytic and geometric definitions, see Section 2.2 for details. But in the Heisenberg group case there exists an additional condition for $K$-quasiconformal mappings $f$. Namely, such an $f$ has to preserve the contact form

$$
\omega=d t+2 \Im(\bar{z} d z)
$$

of $\mathfrak{H}$, i.e., $f^{*} \omega=\lambda \omega$ a.e., for some non-vanishing function $\lambda$. Moreover, if $f=\left(f 1, f_{2}, f_{3}\right)$ then the distributional derivatives $Z f_{I}$ and $Z f_{I}$ exist a.e. Where, $f_{I}=f_{1}+i f_{2}$ and $Z, \bar{Z}$ are the horizontal vector fields

$$
Z=\frac{\partial}{\partial z}+i \bar{z} \frac{\partial}{\partial t}, \quad Z=\frac{\partial}{\partial \bar{z}}-i z \frac{\partial}{\partial t} .
$$

If $f$ is orientation-preserving, then it satisfies a.e. the following Beltrami system of equations:

$$
\bar{Z} f_{I}=\mu_{f} Z f_{I} \quad \text { and } \quad \bar{Z} f_{I I}=\mu_{f} Z f_{I I} .
$$

Here, $f_{I I}=f_{3}+i\left|f_{I}\right|^{2}$ and the Beltrami coefficient $\mu_{f}$ of $f$ is a measurable complex function which is essentially bounded by a constant $k \in[0,1)$, i.e., $\left\|\mu_{f}\right\|_{\infty}=k$. For an arbitrary orientationpreserving homeomorphism $f$ such that $Z f_{I}$ and $\bar{Z} f_{I}$ exist a.e., we consider the Beltrami coefficient, the distortion function of $f$ and the the maximal distortion of $f$ which are given respectively by

$$
\mu_{f}(z, t)=\frac{\bar{Z} f_{I}(z, t)}{Z f_{I}(z, t)}, \quad K_{f}(z, t)=\frac{\left|\mu_{f}(z, t)\right|+1}{\left|\mu_{f}(z, t)\right|-1}, \quad K_{f}=\operatorname{ess} \sup _{(z, t)} K_{f}(z, t) .
$$

For a quasiconformal $f$ we have $\left\|\mu_{f}\right\|_{\infty}=k<1$ and thus $1 \leq K_{f}=\frac{1+k}{1-k}<\infty$. In this paper we consider $\mathcal{C}^{2}$ orientation-preserving contact transformations such that $1 \leq K_{f}<\infty$. These have to be quasiconformal, see Section 2.2,

As in the case of the complex plane, there are two kinds of extremal problems we are interested in: first, we seek for minimisers for the maximal distortion $K_{f}$ among a class $\mathcal{F}$ of sense preserving quasiconformal maps $f: \Omega \rightarrow \Omega^{\prime}$ between domains of $\mathfrak{H}$ which satisfy certain boundary conditions. Standard family arguments and appropriate conditions on $\mathcal{F}$ ensure the existence of a minimiser for the maximal distortion, see Theorem $\mathrm{F}$ in [12] however, there is no standard method to detect 
such a minimiser. For the second case, it turns out that there are two kinds of mean distortions that arise naturally in the Heisenberg group case. First, we have the 2-mean distortion $\mathfrak{M}_{2}(f, \rho)$ of a quasiconformal map $f$ which is given by

$$
\mathfrak{M}_{2}(f, \rho)=\frac{\iiint_{\Omega} K_{f}^{2}(p) \rho^{4}(p) d \mathcal{L}^{3}(p)}{\iiint_{\Omega} \rho^{4}(p) d \mathcal{L}^{3}(p)},
$$

where $\rho: \Omega \rightarrow \mathbb{R}^{+}$is a Borel function related to the geometry of $\Omega$. In [2], Balogh, Fässler and the author developed a method relying on moduli of curve families inside a region of the Heisenberg group, resulting to the following theorem.

Theorem 1.2. For any $k \in(0,1)$, the stretch map $f_{k}$ is a sense preserving quasiconformal map from the Heisenberg spherical ring $S_{a, b}$ onto the Heisenberg spherical ring $S_{a^{k}, b^{k}}$ with maximal distortion $K_{f_{k}}=k^{-2}$. Denote by $\mathcal{F}$ the class of quasiconformal maps $f: S_{a, b} \rightarrow S_{a^{k}, b^{k}}$ which preserve the axis $(0, t)$ and map the boundary components of $S_{a, b}$ to the respective boundary components of $S_{a^{k}, b^{k}}$. Then up to composition with rotations around the vertical axis the stretch map $f_{k}$ minimises the 2-mean distortion within the class $\mathcal{F}$ : for any $f \in \mathcal{F}$ we have

$$
k^{-3}=K_{f_{k}}^{3 / 2}=\mathfrak{M}_{2}\left(f_{k}, \rho_{0}\right) \leq \mathfrak{M}_{2}\left(f, \rho_{0}\right),
$$

where

$$
\rho_{0}(z, t)=\frac{1}{\log (b / a)} \cdot \frac{|z|}{\left.|| z\right|^{2}-i t \mid} \cdot \mathcal{X}\left(S_{a, b}\right)(z, t), \quad \text { for every } \quad(z, t) \in \mathfrak{H} .
$$

Moreover, $K_{f} \geq K_{f_{k}}^{3 / 4}$.

The stretch map $f_{k}$ of Theorem 1.2 (which is actually a shrink map since $k \in(0,1)$ ) is a generalisation of the classical stretch map

$$
g_{k}(z)=z|z|^{k-1}, \quad z \in \mathbb{C}
$$

In cartesian coordinates $f_{k}$ is given by the formula

$$
f_{k}(z, t)=\left(k^{1 / 2} z\left(\frac{\bar{w}}{k|z|^{2}+i t}\right)^{1 / 2}|w|^{\frac{k-1}{2}}, t \cdot \frac{|w|^{k}}{k|z|^{2}+i t}\right),
$$

where $w=|z|^{2}-i t$. Observe that if we set $t=0$ we find $f_{k}(z, 0)=\left(g_{k}(z), 0\right)$ and in this way we recover the classical stretch. Nevertheless, the cartesian expression of $f_{k}$ is rather complicated; the stretch is best described in the parametrisation of $\mathfrak{H}$ by logarithmic coordinates $(\xi, \psi, \eta)$. This parametrisation is given by

$$
(\xi, \psi, \eta) \mapsto\left(i \cos ^{1 / 2} \psi e^{\frac{\xi+i(\psi-3 \eta)}{2}},-\sin \psi e^{\xi}\right),
$$

where $\xi \in \mathbb{R}, \psi \in[-\pi / 2, \pi / 2]$ and $\psi-3 \pi \leq 3 \eta \leq \psi=\pi$ (for details, see Section [5.1 as well as [2] and [18]). Those coordinates are analogous to the logarithmic coordinates of the complex plane; the contact form, the contact conditions and the Beltrami equations admit expressions which are considerably easier to handle than in the cartesian case. In fact, the expression for the stretch $f_{k}$ is

$$
f_{k}(\xi, \psi, \eta)=\left(k \xi, \arctan \left(\frac{\tan \psi}{k}\right), \eta\right) .
$$

Note finally that the above result holds only for $k \in(0,1)$; for $k>1$ the particular modulus method does not work. 
We now define the $2 / 3$-mean distortion $\mathfrak{M}_{2 / 3}\left(f, \rho_{0}\right)$ of a quasiconformal transformation $f$ by

$$
\mathfrak{M}_{2 / 3}\left(f, \rho_{0}\right)=\frac{\iiint_{\Omega} K_{f}^{2 / 3}(p) \rho_{0}^{4 / 3}(p) d \mathcal{L}^{3}(p)}{\iiint_{\Omega} \rho^{4 / 3}(p) d \mathcal{L}^{3}(p)},
$$

where again $\rho_{0}: \Omega \rightarrow \mathbb{R}^{+}$is a Borel function related to the geometry of $\Omega$. In this article we prove the following theorem.

Theorem 1.3. Let $k \in(0,1)$ and denote by $\mathcal{F}^{\prime}$ the class of $\mathcal{C}^{2}$ contact quasiconformal maps $f: S_{a, b} \rightarrow S_{a^{k}, b^{k}}$ which preserve the axis $(0, t)$ and map the boundary components of $S_{a, b}$ to the respective boundary components of $S_{a^{k}, b^{k}}$. Then up to composition with rotations around the vertical axis the stretch map $f_{k}$ minimises the $2 / 3$-mean distortion within the class $\mathcal{F}:$ for any $f \in \mathcal{F}$ we have have

$$
k^{-1 / 3}=K_{f_{k}}^{1 / 6}=\mathfrak{M}_{2 / 3}\left(f_{k}, \rho_{0}\right) \leq \mathfrak{M}_{2 / 3}\left(f, \rho_{0}\right),
$$

where

$$
\rho_{0}(z, t)=\frac{1}{4 \pi \log (b / a)} \cdot \frac{1}{\left.|z| \cdot|| z\right|^{2}-i t \mid} \cdot \mathcal{X}\left(S_{a, b}\right)(z, t) \quad \text { for every } \quad(z, t) \in \mathfrak{H} .
$$

Moreover, $K_{f} \geq K_{f_{k}}^{1 / 4}$.

To prove this theorem we develop a method of modulus of surfaces as a counterpart of the modulus method of curve families that is developed in [2]. Throughout this paper, a surface $\mathcal{S}$ inside $\mathfrak{H}$ is supposed in the Euclidean sense and endowed with sufficient regularity (i.e., $\mathcal{C}^{2}$ ). Outside an exceptional set of $\mathcal{S}$ of small Lebesgue measure (the characteristic locus of $\mathcal{S}$ ), a horizontal vector field along $\mathcal{S}$ is canonically defined; this is the horizontal normal vector field $N_{\mathcal{S}}^{h}$ of $\mathcal{S}$. That is, the vector field $N_{\mathcal{S}}^{h}$ of $\mathcal{S}$ lies in the horizontal subbubdle of $\mathfrak{H}$ at points of $\mathcal{S}$. The following property (see Proposition 3.13) holds for surfaces $\mathcal{S}$ and $\widetilde{\mathcal{S}}$ such that $\widetilde{\mathcal{S}}=f(\mathcal{S})$ where $f=\left(f_{I}, f_{3}\right)$ is a $\mathcal{C}^{2}$ contact quasiconformal transformation:

$$
\lambda\left(\left|Z f_{I}\right|-\left|\bar{Z} f_{I}\right|\right) \cdot\left\|N_{\mathcal{S}}^{h}\right\| \leq\left\|N_{\widetilde{\mathcal{S}}}^{h}\right\| \leq \lambda\left(\left|Z f_{I}\right|+\left|\bar{Z} f_{I}\right|\right) \cdot\left\|N_{\mathcal{S}}^{h}\right\|
$$

Where, $\lambda$ is the square root of the Jacobian $J_{f}$ of $f$. A transformation $f$ is said to have the minimal stretching property $(m S P)$ for $\mathcal{S}$ if the left hand side inequality is attained as an equality.

Given now a family $\Sigma$ of such surfaces inside a domain $\Omega \subset \mathfrak{H}$, we define in Section 4.1 the $\operatorname{modulus} \operatorname{Mod}(\Sigma)$ as

$$
\operatorname{Mod}(\Sigma)=\inf _{\rho \in \operatorname{Adm}(\Sigma)} \iiint_{\Omega} \rho^{4 / 3} d \mathcal{L}^{3}
$$

Here, $\operatorname{Adm}(\Sigma)$ is the set of positive Borel functions $\rho$ defined in $\Omega$ such that

$$
\iint_{\mathcal{S}} \rho d S^{h} \geq 1
$$

The integral on the left is defined in a local parametrisation $\sigma: U \rightarrow \mathbb{R}^{3},(u, v) \mapsto \sigma(u, v)$ as

$$
\iint_{U} \rho(\sigma(u, v))\left\|N_{\sigma}^{h}(u, v)\right\| d u d v
$$

The following Modulus Inequality (Theorem 4.2) holds: if $f: \Omega \rightarrow \Omega^{\prime}$ is a $\mathcal{C}^{2}$ contact quasiconformal transformation between domains of $\mathfrak{H}$ with distortion function $K_{f}(p)$ and $\Sigma$ is a family of surfaces inside $\Omega$ then

$$
\operatorname{Mod}(f(\Sigma)) \leq \iiint_{\Omega} K_{f}^{2 / 3}(p) \rho^{4 / 3}(p) d \mathcal{L}^{3}(p)
$$


for every $\rho \in \operatorname{Adm}(\Sigma)$. From this, we also obtain that for an extremal density $\rho_{0}$ of $\Sigma$, i.e., for a $\rho_{0} \in \operatorname{Adm}(\Sigma)$ such that $\operatorname{Mod}(\Sigma)=\iiint_{\Omega} \rho_{0}^{4 / 3} d \mathcal{L}^{3}$ we have

$$
\frac{\operatorname{Mod}(f(\Sigma))}{\operatorname{Mod}(\Sigma)} \leq \mathfrak{M}_{2 / 3}\left(f, \rho_{0}\right)
$$

and also, if $K_{f}$ is the maximal distortion of $f$ then

$$
K_{f}^{-2 / 3} \operatorname{Mod}(\Sigma) \leq \operatorname{Mod}(f(\Sigma)) \leq K_{f}^{2 / 3} \operatorname{Mod}(\Sigma) .
$$

The modulus method we develop here concerns domains $\Omega$ of $\mathfrak{H}$ which are foliated in a particular way by a family $\Sigma_{0}$ of surfaces inside $\Omega$, see Lemma 4.4; in this case we find an extremal density $\rho_{0}$ for $\Sigma_{0}$ from which we may explicitly calculate $\operatorname{Mod}\left(\Sigma_{0}\right)$. Let now $\mathcal{F}^{\prime}$ be the class of all $\mathcal{C}^{2}$ contact quasiconformal maps from $\Omega$ to another domain $\Omega^{\prime}$ which satisfy certain boundary conditions. Suppose additionally that there exists an $f_{0} \in \mathcal{F}^{\prime}$ which has the mSP for each surface of $\Sigma_{0}$ and is also such that its distortion function $K_{f_{0}}(p)$ is constant on each leaf $\mathcal{S} \in \Sigma_{0}$, see Lemma 4.5. Then, we can calculate $\operatorname{Mod}\left(f_{0}\left(\Sigma_{0}\right)\right)$ and show that it equals to $\iiint_{\Omega} K_{f_{0}}^{2 / 3}(p) \rho_{0}^{4 / 3}(p) d \mathcal{L}^{3}(p)$. In summary, our modulus method is given in the next theorem.

Theorem 1.4. Let $\Sigma_{0}, \rho_{0}$ and $f_{0}$ be such that they satisfy the assumptions of Lemmas 4.4 and 4.5. Let $\Sigma \supseteq \Sigma_{0}$ be a family of $\mathcal{C}^{2}$ surfaces in $\Omega$ so that $\rho_{0} \in \operatorname{Adm}(\Sigma)$ and consider the class $\mathcal{F}^{\prime}$ of all $\mathcal{C}^{2}$ contact quasiconformal transformations $f: \Omega \rightarrow \Omega^{\prime}$ which satisfy

$$
\operatorname{Mod}\left(f_{0}\left(\Sigma_{0}\right)\right) \leq \operatorname{Mod}(f(\Sigma))
$$

Then for all $f \in \mathcal{F}^{\prime}$ we have

$$
\mathfrak{M}_{2 / 3}\left(f_{0}, \rho_{0}\right) \leq \mathfrak{M}_{2 / 3}\left(f, \rho_{0}\right)
$$

In the proof of Theorem 1.3 our family $\Sigma_{0}$ consists of Heisenberg cones $\mathcal{C}_{\alpha}$, i.e., paraboloids with cartesian equation $t=-\alpha|z|^{2}$. These surfaces foliate $S_{a, b}$ in a manner according to the assumptions of Lemma 4.4, subsequently, in Proposition 5.1 we find the density $\rho_{0}$ as in Theorem 1.3 and also that

$$
\operatorname{Mod}\left(\Sigma_{0}\right)=\left(2^{5} \pi \log (b / a)\right)^{-1 / 3} \mathrm{~B}(1 / 2,1 / 6) .
$$

Now, for $k \in(0,1)$ the stretch map $f_{k}$ satisfies the assumptions of Lemma 4.5, i.e., it has the mSP for $\Sigma_{0}$ and it is constant at each leaf. Thus we find

$$
\operatorname{Mod}\left(f_{k}\left(\Sigma_{0}\right)\right)=\iiint_{S_{a, b}} K_{f_{k}}^{2 / 3}(p) \rho_{0}^{4 / 3}(p) d \mathcal{L}^{3}(p)=k^{-1 / 3} \cdot \operatorname{Mod}\left(\Sigma_{0}\right) .
$$

In order to apply Theorem 1.4 and conclude the proof, we must detect a surface family $\Sigma$ such that the density $\rho_{0}$ is admissible for $\Sigma$ and $\left.f_{k}\left(\Sigma_{0}\right)\right) \subseteq f(\Sigma)$ for each $f \in \mathcal{F}^{\prime}$. Indeed, we find that this family $\Sigma$ consists of surfaces inside $S_{a, b}$ which join the two pieces of the boundary and enjoy an additional property: for every $\mathcal{S} \in \Sigma$, there is no non-characteristic point $p \in \mathcal{S}$ with a neighborhood $U_{p}$ being a piece of a Heisenberg sphere.

At this point, we wish to make the following comment on the general setup of this work and in particular, on the proof of our main theorem. Having assumed $\mathcal{C}^{2}$ regularity for our surfaces lead us to find a minimiser of the 2/3-mean distortion $\mathfrak{M}_{2 / 3}\left(f, \rho_{0}\right)$ only among the class $\mathcal{F}^{\prime}$ of $\mathcal{C}^{2}$ contact quasiconformal mappings $f$ between spherical rings $S_{a, b}$ and $S_{a^{k}, b^{k}}$ of $\mathfrak{H}$ which map boundary components to respective boundary components. Of course, this class is smaller than the class $\mathcal{F}$ of arbitrary quasiconformal transformations between these two domains which satisfy the same boundary conditions. It seems plausible to conjecture that the same method may apply in the 
case of $\mathfrak{H}$-regular surfaces (for the definition and the properties of these surfaces, see for instance [11]). This will enable us to omit the regularity hypothesis for the quasiconformal mappings of our theorem and prove in fact that the stretch map is a minimiser within the wider class $\mathcal{F}$.

The paper is organised as follows. Preliminaries about the Heisenberg group $\mathfrak{H}$ and quasiconformal mappings defined on $\mathfrak{H}$ are given in Section 2 . Section 3 is brief study of $\mathcal{C}^{2}$ regular surfaces embedded in $\mathfrak{H}$. The definition of the modulus of a surface family, the Modulus Inequality as well as the modulus method and the proof of Theorem 1.4 are in Section 4. Finally, the proof of our main Theorem 1.3 lies in Section 5 .

Aknowledgments. The author wishes to thank Zoltán Balogh and Katrin Fässler for fruitful discussions and useful observations.

\section{Preliminaries}

The material of this section is standard and we refer the reader to 4 for more details. The Heisenberg group is described in Section 2.1 and a brief overview of the Korányi-Reimann theory of quasiconformal mappings of $\mathfrak{H}$ lies in Section 2.2 .

2.1. Heisenberg Group. The Heisenberg group $\mathfrak{H}$ is $\mathbb{C} \times \mathbb{R}$ whose group law and distance $d_{\mathfrak{H}}$ are described in the introduction. The metric $d_{\mathfrak{H}}$ is invariant by a left translations $T_{(\zeta, s)}$, defined for a given $(\zeta, s) \in \mathfrak{H}$ by $T_{(\zeta, s)}(z, t)=(\zeta, s) *(z, t)$ and also by rotations about the vertical axis $R_{\theta}$, defined

for a given $\theta \in \mathbb{R}$ by $R_{\theta}(z, t)=\left(z e^{i \theta}, t\right)$. Left translations and rotations form the group $\operatorname{Isom}\left(\mathfrak{H}, d_{\mathfrak{H}}\right)$ of (orientaion-preserving) Heisenberg isometries. There are two other kinds of transformations that are of particular importance; in the first place we have dilations $D_{\delta}$ defined for a given $\delta>0$ by $D_{\delta}(z, t)=\left(\delta z, \delta^{2} t\right)$. One may check that the metric $d_{\mathfrak{H}}$ is scaled up to the multiplicative constant $\delta$ by the action of $D_{\delta}$. Finally there is an inversion $I$ with respect to the unit Heisenberg sphere given for all $(z, t) \neq(0,0)$ by $I(z, t)=\left(\frac{z}{-|z|^{2}+i t}, \frac{-i t}{\left.|-| z\right|^{2}+\left.i t\right|^{2}}\right)$.

The Heisenberg group $\mathfrak{H}$ is a 2 -step nilpotent Lie group with left invariant vector fields

$$
X=\frac{\partial}{\partial x}+2 y \frac{\partial}{\partial t}, \quad Y=\frac{\partial}{\partial y}-2 x \frac{\partial}{\partial t}, \quad T=\frac{\partial}{\partial t}
$$

and the complex fields $Z, \bar{Z}$ as in the introduction are just

$$
Z=\frac{1}{2}(X-i Y), \quad \bar{Z}=\frac{1}{2}(X+i Y) .
$$

There is a non-trivial commutation relation $[X, Y]=-4 T$ and the tangent space to $\mathfrak{H}$ is spanned by $X, Y, T$. The Lie algebra of left invariant vector fields of $\mathfrak{H}$ has a grading $\mathfrak{h}=\mathfrak{v}_{1} \oplus \mathfrak{v}_{2}$ with

$$
\mathfrak{v}_{1}=\operatorname{span}_{\mathbb{R}}\{X, Y\} \quad \text { and } \quad \mathfrak{v}_{2}=\operatorname{span}_{\mathbb{R}}\{T\} .
$$

The contact form $\omega$ of $\mathfrak{H}$ is defined as the unique 1 -form satisfying $X, Y \in \operatorname{ker} \omega, \omega(T)=1$; in $(z, t)$ coordinates

$$
\omega=d t+2 \Im(\bar{z} d z) .
$$

Uniqueness here is modulo change of coordinates as it follows by the Darboux's Theorem. The distribution $\mathrm{H}(\mathfrak{H})=\mathfrak{v}_{1}$ is called the horizontal distribution, any vector field $V \in \mathfrak{v}_{1}$ is called a horizontal vector field and if $p \in \mathfrak{H}$, the $\operatorname{space}_{p}(\mathfrak{H})=\operatorname{span}_{\mathbb{R}}\left\{X_{p}, Y_{p}\right\}$ is called the horizontal tangent plane to $p$. The sub-Riemannian metric of $\mathfrak{H}$ is the metric defined in $\mathrm{H}(\mathfrak{H})$ by the relations $\langle X, X\rangle=\langle Y, Y\rangle=1$ and $\langle X, Y\rangle=\langle Y, X\rangle=0$; its norm shall be denoted by $\|\cdot\|$. 
The Legendrian foliation is the foliation of $\mathfrak{H}$ by horizontal curves. An absolutely continuous curve $\gamma:[a, b] \rightarrow \mathfrak{H}$ (in the Euclidean sense) with $\gamma(t)=\left(\gamma_{I}(t), \gamma_{3}(t)\right) \in \mathbb{C} \times \mathbb{R}$ is called horizontal if

$$
\dot{\gamma}(t) \in \mathrm{H}_{\gamma(t)}(\mathfrak{H}) \quad \text { for almost every } t \in[a, b] .
$$

A curve $\gamma:[a, b] \rightarrow \mathfrak{H}$ is absolutely continuous with respect to the $d_{\mathfrak{H}}$ if and only if it is a horizontal curve.

A contact transformation $f: \Omega \rightarrow \Omega^{\prime}$ on $\mathfrak{H}$ is a diffeomorphism between domains $\Omega$ and $\Omega^{\prime}$ in $\mathfrak{H}$ which preserves the contact structure, i.e.,

$$
f^{*} \omega=\lambda \omega,
$$

for some non-vanishing real valued function $\lambda$. We write $f=\left(f_{I}, f_{3}\right), f_{I}=f_{1}+\mathrm{i} f_{2}$. Then a contact map $f$ is completely determined by $f_{I}$ in the sense that the contact condition (2.1) is equivalent to the following system of differential equations:

$$
\begin{aligned}
& \bar{f}_{I} Z f_{I}-f_{I} Z \bar{f}_{I}+i Z f_{3}=0 \\
& f_{I} \bar{Z} \bar{f}_{I}-\bar{f}_{I} \bar{Z} f_{I}-i \bar{Z} f_{3}=0 \\
& -i\left(\bar{f}_{I} T f_{I}-f_{I} T \bar{f}_{I}+i T f_{3}\right)=\lambda .
\end{aligned}
$$

If $f$ is $\mathcal{C}^{2}$ then $\operatorname{det} J_{f}=\lambda^{2}$, where by $J_{f}$ we denote the usual Jacobian matrix of $f$.

2.2. Quasiconformal Mappings. There are various analytic definitions of quasiconformality in $\mathfrak{H}$ which are all equivalent to the metric definition [1.1; for instance, we refer the reader to [5], [10] and [23]. For the analytic definition of qc mappings we state here, we follow the lines of [12] and [13] with minor deviations. We first recall the notions of the P-differentiability of mappings between domains of $\mathfrak{H}$ and that of the absolute continuity in lines (ACL). According to Pansu, [16], a mapping $f: \Omega \rightarrow \Omega^{\prime}$ between domains of $\mathfrak{H}$ is called $P$-differentiable at $p \in \Omega$ if for $c \rightarrow 0$ the mappings

$$
D_{c}^{-1} \circ T_{f(p)}^{-1} \circ f \circ T_{p} \circ D_{c}
$$

converge locally uniformly to a homomorphism $\left(D_{0}\right) f_{p}$ from $T_{p}(\mathfrak{H})$ to $T_{f(p)}(\mathfrak{H})$ which preserves the horizontal space $\mathrm{H}(\mathfrak{H})$. Here $D$ and $T$ are dilations and left translations respectively. In terms of the standard basis $Z, \bar{Z}, T$, the $P$-derivative of $f=\left(f_{I}, f_{3}\right)$ at $p$ is in matrix form

$$
\left(D_{0}\right) f_{p}=\left(\begin{array}{ccc}
Z f_{I} & \bar{Z} f_{I} & 0 \\
Z \overline{f_{I}} & \overline{Z f_{I}} & 0 \\
0 & 0 & \left|Z f_{I}\right|^{2}-\left|\bar{Z} f_{I}\right|^{2}
\end{array}\right)_{p},
$$

where all derivatives are in the distributional sense. Quasiconformal mappings between domains in $\mathfrak{H}$ are a.e. $P$-differentiable, see [16. In particular, (see Proposition 6 of [13]), if $f$ is $P$-differentiable at $p \in \mathfrak{H}$ with derivative $\left(D_{0}\right) f_{p}$, then the restriction of $f$ to the plane

$$
\left\{p \exp (x X+y Y) \mid(x, y) \in \mathbb{R}^{2}\right\}
$$

is differentiable at $p$ in the Euclidean sense and its derivative $\left(D_{h}\right) F_{p}$ is the restriction of $\left(D_{0}\right) F_{p}$ in horizontal spaces; in matrix form, it is given by

$$
\left(D_{h}\right) f_{p}=\left(\begin{array}{ll}
Z f_{I} & \bar{Z} f_{I} \\
Z f_{I} & \overline{Z f_{I}}
\end{array}\right)_{p}
$$

and the following also hold for a $K$-quasiconformal mapping $f$ which is $P$-differentiable at $p$ :

(1) $\left\|\left(D_{h}\right) f_{p}\right\|:=\max \left\{\left\|\left(D_{h}\right) f_{p}(V)\right\| \mid\|V\|=1\right\}=\left|Z f_{I}(p)\right|+\left|\bar{Z} f_{I}(p)\right|$ a.e.;

(2) $J_{f}(p)=\operatorname{det}\left(D_{0}\right) f_{p}=\left(\operatorname{det}\left(D_{h}\right) f_{p}\right)^{2}=\left(\left|Z f_{I}(p)\right|^{2}-\left|\bar{Z} f_{I}(p)\right|^{2}\right)^{2}$; 
(3)

$$
K_{f}(p)^{2}=\frac{\left\|\left(D_{h}\right) f_{p}\right\|^{4}}{J_{f}(p)}=\left(\frac{\left|Z f_{I}(p)\right|+\left|\bar{Z} f_{I}(p)\right|}{\left|Z f_{I}(p)\right|-\left|\bar{Z} f_{I}(p)\right|}\right)^{2} \leq K .
$$

The function $\Omega \ni p \rightarrow K_{f}(p) \in[1, \infty)$ is the distortion function of $f$ and the constant $K_{f}=K^{1 / 2}$ is also called the maximal distortion of $f$.

A mapping between domains in the Heisenberg group $\mathfrak{H}$ is called absolutely continuous in lines (ACL), if it is absolutely continuous on almost all fibers of smooth horizontal fibrations. For such a fibration, the fibers $\gamma_{p}$ can be parametrised by the flow $f_{s}$ of a horizontal unit vector field $V$ : i.e., $V$ is of the form $a X+b Y$ with $|a|^{2}+|b|^{2}=1$. Mostow proved (see Theorem A in [13]) that quasiconformal mappings are absolutely continuous on a.e. fiber $\gamma$ of any give fibration $\Gamma_{V}$ determined by a left invariant horizontal vector field $V$.

Beltrami equations are in order. According to Theorem $\mathrm{C}$ in [13], if $f=\left(f_{I}, f_{3}\right)$ is an orientation preseving $K$-quasiconformal mapping between domains $\Omega$ and $\Omega^{\prime}$ in $\mathfrak{H}$ then it satisfies a.e. the Beltrami type system of equations

$$
\begin{aligned}
& \bar{Z} f_{I}=\mu Z f_{I}, \\
& \bar{Z} f_{I I}=\mu Z f_{I I},
\end{aligned}
$$

where $f_{I I}=f_{3}+i\left|f_{I}\right|^{2}$ and $\mu$ is a complex function in $\Omega$ such that

$$
\frac{1+\|\mu\|_{\infty}}{1-\|\mu\|_{\infty}} \leq K \quad \text { a.e. }
$$

where $\|\mu\|_{\infty}=\operatorname{esssup}\{|\mu(z, t)| \mid(z, t) \in \Omega\}$. For each $p=(z, t) \in \Omega$, the function

$$
\mu(p)=\mu_{f}(p)=\frac{\bar{Z} f_{I}(p)}{Z f_{I}(p)},
$$

is called the Beltrami coefficient of $f$. If $K_{f}$ is the maximal distortion and $K_{f}(p)$ is the distortion function of $f$ respectively, then the following hold:

$$
\left|\mu_{f}(p)\right|=\frac{K_{f}(p)-1}{K_{f}(p)+1}, \quad K_{f}(p)=\frac{1+\left|\mu_{f}(p)\right|}{1-\left|\mu_{f}(p)\right|}, \quad\left\|\mu_{f}\right\|_{\infty}=\frac{K_{f}-1}{K_{f}+1} .
$$

We now state the analytic definition of quasiconformality in $\mathfrak{H}$.

Definition 2.1. (Analytic definition) A homeomorphism $f: \Omega \rightarrow \Omega^{\prime}, f=\left(f_{I}, f_{3}\right)$, between domains in $\mathfrak{H}$ is an orientation-preserving quasiconformal mapping if

(i) it is ACL;

(ii) it is a.e. $P$-differentiable, and

(iii) it satisfies a.e. a system of Beltrami equations of the form 2.5, 2.6 where $\mu$ is a complex function in $\Omega$ such that $\|\mu\|_{\infty}<1$.

An analogous definition holds for orientation reserving quasiconformal mappings.

In the present paper we are considering quasiconformal maps with sufficient smoothness; these have to be contact transformations. From $P$-differentiability of quasiconformal maps it follows that $P$-diffeomorphic $K$-quasiconformal mappings are contact transformations satisfying

$$
\left\|\left(D_{h}\right) f\right\|^{4} \leq K\left|J_{f}\right| \text { a.e. }
$$

(Here, the absolute value in the Jacobian covers both situations of orientation-preserving and orientation-reversing mappings). The converse is also true, see Proposition 8 in [13]: if a $\mathcal{C}^{2}$ 
contact transformation $f$ satisfies condition (2.7), then $f$ is $K$-quasiconformal. We conclude that $K$-quasiconformal diffeomorphism lie in the class of contact transformations. Due to the contact conditions (2.2), (2.3) and (2.4), equation (2.5) in the Beltrami system implies equation (2.6).

\section{Surfaces in the Heisenberg Group}

There is a rather large bibliography about surfaces in the Heisenberg group and their geometrical properties; the reader should see for instance [4] for a treatment of hypersurfaces of $\mathfrak{H}$ and the references given therein. Our treatment in this section is somewhat different, see [17]; instead of hypersurfaces, i.e., graphs of function with sufficient regularity, we study regular surfaces $\mathcal{S}$ (here, regular means $\mathcal{C}^{2}$ regular in the Euclidean sense) via surface patches. Accordingly, we define the horizontal space $\mathbb{H}(\mathcal{S})$, the horizontal normal $N_{\mathcal{S}}^{h}$ and the characteristic locus $\mathfrak{C}(\mathcal{S})$ of $\mathcal{S}$ in Section 3.1. In Section 3.2 we show that the pullback of the contact form $\omega$ of $\mathfrak{H}$ in a regular surface $\mathcal{S}$ defines a contact 1-form $\omega_{\mathcal{S}}$ on $\mathcal{S}$ whose kernel is $\mathbb{J} N_{\mathcal{S}}^{h}$; here $\mathbb{J}$ is the natural complex operator acting on $\mathbb{H}(\mathcal{S})$. The integral curves of $\mathbb{J} N_{\mathcal{S}}^{h}$ are horizontal curves lying in $\mathcal{S}$. Next, the horizontal area of a regular surface $\mathcal{S}$ is defined in Section 3.3. The definition here is via surface patches and in the case of hypersurfaces it agrees with the definition given in [4]. Finally, Section [3.4] is devoted to the study of contact $\mathcal{C}^{2}$ transformations of $\mathfrak{H}$ which map a regular surface to another. Proposition 3.13 and Corollary 3.14 are crucial for our subsequent discussion.

3.1. Regular Surfaces, Horizontal Normal. For clarity, we recall the notion of regular surface of $\mathbb{R}^{3}$ (see, for instance, [6]): this is a countable collection of surface patches (local charts) $\sigma_{\alpha}: U_{\alpha} \rightarrow V_{\alpha} \cap \mathbb{R}^{3}$ where $U_{\alpha}$ and $V_{\alpha}$ are open sets of $\mathbb{R}^{2}$ and $\mathbb{R}^{3}$, respectively, such that

(1) each $\sigma_{\alpha}$ is a $\mathcal{C}^{2}$ homeomorphism, and

(2) the differential $\left(\sigma_{\alpha}\right)_{*}: \mathbb{R}^{2} \rightarrow \mathbb{R}^{3}$ is of rank 2 everywhere.

The tangent plane $T_{\sigma}(\mathcal{S})$ of $\mathcal{S}$ at a surface patch $\sigma$ defined in an open domain $U \subset \mathbb{R}^{2}$ by

$$
\sigma(u, v)=(x(u, v), y(u, v), t(u, v))
$$

is $T_{\sigma}(\mathcal{S})=\operatorname{span}\left\{\sigma_{u}=\sigma_{*} \frac{\partial}{\partial u}, \sigma_{v}=\sigma_{*} \frac{\partial}{\partial v}\right\}$. This may also be defined by the normal vector

$$
N_{\sigma}=\sigma_{u} \wedge \sigma_{v}=\frac{\partial(y, t)}{\partial(u, v)} \frac{\partial}{\partial x}+\frac{\partial(t, x)}{\partial(u, v)} \frac{\partial}{\partial y}+\frac{\partial(x, y)}{\partial(u, v)} \frac{\partial}{\partial t},
$$

where $\wedge$ is the exterior product in $\mathbb{R}^{3}$. We have $T_{\sigma}(\mathcal{S})=\left\{V_{\sigma} \in T_{\sigma}\left(\mathbb{R}^{3}\right): N_{\sigma} \cdot V_{\sigma}=0\right\}$ where the dot is the usual Euclidean product in $\mathbb{R}^{3}$.

A regular surface $\mathcal{S}$ is oriented if for every two overlapping patches $(U, \sigma)$ and $(\tilde{U}, \tilde{\sigma})$ the transition mapping $\Phi=\sigma^{-1} \circ \tilde{\sigma}$ has positive Jacobian deterninant $J_{\Phi}=\operatorname{det}\left(\Phi_{*}\right)$. In $U \cap \tilde{U}$ we then have $N_{\tilde{\sigma}}^{h}=J_{\Phi} N_{\sigma}^{h}$ and the unit normal vector field of $\nu_{\mathcal{S}}$ of $\mathcal{S}$ is uniquely defined at each local chart by the relation

$$
\nu_{\sigma}=\frac{\sigma_{u} \wedge \sigma_{v}}{\left|\sigma_{u} \wedge \sigma_{v}\right|}
$$

where $|\cdot|$ is the Euclidean norm in $\mathbb{R}^{3}$.

From now on, by a regular surface in $\mathfrak{H}$ we shall always mean a regular oriented surface in $\mathbb{R}^{3}$.

Definition 3.1. Let $\mathcal{S}$ be a regular surface and $p \in \mathcal{S}$. The horizontal plane $\mathbb{H}_{p}(\mathcal{S})$ of $\mathcal{S}$ at $p$ is the horizontal plane $\mathbb{H}_{p}(\mathfrak{H})$. 
Next, we define the horizontal normal vector $N_{p}^{h}$ at an arbitrary $p \in \mathcal{S}$. To do so, we first define the Heisenberg wedge product $\wedge_{p}^{\mathfrak{H}}$; this is the linear mapping $T_{p}(\mathfrak{H}) \times T_{p}(\mathfrak{H}) \rightarrow T_{p}(\mathfrak{H})$ which assigns to each two vectors $a, b \in T_{p}(\mathfrak{H})$, where $a=a_{1} X+a_{2} Y+a_{3} T$ and $b=b_{1} X+b_{2} Y+b_{3} T$, the vector $a \wedge^{\mathfrak{H}} b \in T_{p}(\mathfrak{H})$ which is given by the formal determinant

$$
a \wedge^{\mathfrak{H}} b=\left|\begin{array}{ccc}
X & Y & T \\
a_{1} & a_{2} & a_{3} \\
b_{1} & b_{2} & b_{3}
\end{array}\right|=\left|\begin{array}{cc}
a_{2} & a_{3} \\
b_{2} & b_{3}
\end{array}\right| X+\left|\begin{array}{cc}
a_{3} & a_{1} \\
b_{3} & b_{1}
\end{array}\right| Y+\left|\begin{array}{cc}
a_{1} & a_{2} \\
b_{1} & b_{2}
\end{array}\right| T
$$

One may check that $\wedge_{p}^{\mathfrak{H}}$ is skew-symmetric and that following clock rule holds.

$$
X \wedge^{\mathfrak{H}} Y=T, \quad Y \wedge^{\mathfrak{H}} T=X, \quad T \wedge^{\mathfrak{H}} X=Y .
$$

Definition 3.2. If $\sigma: U \rightarrow \mathbb{R}^{3}$ is a surface patch of a regular surface $\mathcal{S}$, the horizontal normal $N_{\sigma}^{h}$ to $\sigma$ is the horizontal part of $\sigma_{u} \wedge^{\mathfrak{H}} \sigma_{v}=\sigma_{*} \partial / \partial u \wedge^{\mathfrak{H}} \sigma_{*} \partial / \partial v$, that is,

$$
N_{\sigma}^{h}=\left(\sigma_{u} \wedge^{\mathfrak{H}} \sigma_{v}\right)^{h}=\sigma_{u} \wedge^{\mathfrak{H}} \sigma_{v}-\omega\left(\sigma_{u} \wedge^{\mathfrak{H}} \sigma_{v}\right) T .
$$

We stress here that the horizontal normal $N_{\sigma}^{h}$ is not the horizontal part of the normal $N_{\sigma}$. Simple calculations induce the following explicit formula:

$$
N_{\sigma}^{h}=\left(\frac{\partial(y, t)}{\partial(u, v)}+2 y \frac{\partial(x, y)}{\partial(u, v)}\right) X+\left(\frac{\partial(t, x)}{\partial(u, v)}-2 x \frac{\partial(x, y)}{\partial(u, v)}\right) Y .
$$

The horizontal normal $N_{p}^{h}$ at a point $p \in \mathcal{S}$ depends on the choice of the surface patch in the following way: suppose that $(U, \sigma)$ and $(\tilde{U}, \tilde{\sigma})$ are two overlapping patches at $p$. Then if $\Phi=\sigma^{-1} \circ \tilde{\sigma}$ is the transition mapping, we may find from (3.1) that around $p$ we have $N_{\tilde{\sigma}}^{h}=J_{\Phi} N_{\sigma}^{h}$, where $J_{\Phi}=\operatorname{det}\left(\Phi_{*}\right)>0$ since $\mathcal{S}$ is oriented.

Definition 3.3. Let $\mathcal{S}$ be a regular surface. A point $p \in \mathcal{S}$ is called non-characteristic if $N_{p}^{h} \neq 0$. The set of characteristic points $\mathfrak{C}(\mathcal{S})=\left\{p \in \mathcal{S}: N_{p}^{h}=0\right\}$ is called the characteristic locus of $\mathcal{S}$.

By definition, the points of $\mathfrak{C}(\mathcal{S})$ are given in a local chart $(U, \sigma)$ by the equations

$$
\frac{\partial(y, t)}{\partial(u, v)}+2 y \frac{\partial(x, y)}{\partial(u, v)}=0 \quad \text { and } \quad \frac{\partial(t, x)}{\partial(u, v)}-2 x \frac{\partial(x, y)}{\partial(u, v)}=0 .
$$

An equivalent, but independent of coordinates definition of the characteristic locus is given in Proposition 3.5 below.

If $\sigma$ is a surface patch of $\mathcal{S}$ then the unit horizontal normal $\nu_{\sigma}^{h}$ to $\sigma$ is defined at non-characteristic points by

$$
\nu_{\sigma}^{h}=\frac{N_{\sigma}^{h}}{\left\|N_{\sigma}^{h}\right\|}=\frac{\left(\sigma_{u} \wedge^{\mathfrak{H}} \sigma_{v}\right)^{h}}{\left\|\left(\sigma_{u} \wedge^{\mathfrak{H}} \sigma_{v}\right)^{h}\right\|},
$$

where $\|\cdot\|$ denotes the norm of the product $\langle\cdot, \cdot\rangle$ in $\mathfrak{H}($ recall that $\|X\|=\|Y\|=1$ and $\langle X, Y\rangle=0$ ).

Corollary 3.4. Let $\mathcal{S}$ be a regular surface of $\mathfrak{H}$. Then away from the characteristic locus, (3.3) defines a nowhere vanishing vector field $\nu_{\mathcal{S}}^{h} \in \mathbb{H}(\mathcal{S})$, such that $\left\|\nu_{\mathcal{S}}^{h}\right\|=1$. 
Associated to the horizontal normal vector field $\nu_{\mathcal{S}}^{h}$ there is a horizontal vector field induced by the complex operator $\mathbb{J}$ acting in $\mathbb{H}(\mathfrak{H})$ by the relations $\mathbb{J} X=Y$ and $\mathbb{J} Y=-X$. Restricting this action in the horizontal space of a regular surface $\mathcal{S}$, if $\nu_{\mathcal{S}}^{h}=\nu_{1} X+\nu_{2} Y$ then we have

$$
\mathbb{J} \nu_{\mathcal{S}}^{h}=-\nu_{2} X+\nu_{1} Y
$$

3.2. Induced 1-form, Local Contactomorphisms, Horizontal Flow. If $\mathcal{S}$ is a regular surface in $\mathfrak{H}$ then a 1 -form $\omega_{\mathcal{S}}$ may be defined in $\mathcal{S}$ in the following manner. Denote by $\iota_{\mathcal{S}}$ the inclusion map $\iota_{\mathcal{S}}: \mathcal{S} \hookrightarrow \mathfrak{H}$, given locally by a parametrisation $\sigma(u, v)=(x(u, v), y(u, v), t(u, v))$. If $\omega=d t+2 x d y-2 y d x$ is the contact form of $\mathfrak{H}$ then $\omega_{\mathcal{S}}=\iota_{\mathcal{S}}^{*} \omega$; in the local parametrisation this is given by

$$
\omega_{\mathcal{S}}=\sigma^{*} \omega=\left(t_{u}+2 x y_{u}-2 y x_{u}\right) d u+\left(t_{v}+2 x y_{v}-2 y x_{v}\right) d v
$$

We leave the proof of the following proposition to the reader.

Proposition 3.5. The characteristic locus $\mathfrak{C}(\mathcal{S})$ is the (closed) set of points of $\mathcal{S}$ at which $\omega_{\mathcal{S}}=0$.

Definition 3.6. Let $f: \mathcal{S} \rightarrow \tilde{\mathcal{S}}$ be a smooth diffeomorphism between regular surfaces $\mathcal{S}$ and $\tilde{\mathcal{S}}$ and outside the characteristic loci of $\mathcal{S}$ and $\tilde{\mathcal{S}}$. The mapping $f$ is called a local contactomorphism of $\mathcal{S}$ and $\tilde{\mathcal{S}}$ if there exists a smooth function $\lambda$ so that $f^{*} \omega_{\tilde{\mathcal{S}}}=\lambda \omega_{\mathcal{S}}$.

Since $f$ is a local diffeomorphism, if $\sigma: U \rightarrow \mathbb{R}^{3}$ is a surface patch for $\mathcal{S}$ then $\tilde{\sigma}=f \circ \sigma$ is a surface patch for $\tilde{\mathcal{S}}$ (with the possible exception of characteristic points). It follows that $f: \mathcal{S} \rightarrow \tilde{\mathcal{S}}$ is a contactomorphism if and only if

$$
\omega_{\tilde{\sigma}}(u, v)=\lambda(u, v) \omega_{\sigma}(u, v), \quad \text { for almost all }(u, v) \in U .
$$

Let now $\gamma: I \rightarrow \mathcal{S}$ be a surface curve on a regular surface $\mathcal{S}$, that is a smooth mapping from an open interval of $\mathbb{R}$ to $\mathcal{S}$. The following proposition gives the condition under which a surface curve is horizontal, i.e., its horizontal tangent $\dot{\gamma}^{h}(s) \in \mathbb{H}_{\gamma(s)}(\mathcal{S})$.

Proposition 3.7. Suppose that $\sigma: U \rightarrow \mathfrak{H}$ is a surface patch and $\gamma(s)=\sigma(u(s), v(s)), s \in I$, is a smooth surface curve (that is, $\tilde{\gamma}(s)=(u(s), v(s)$ ) a smooth curve in $U$ ). Then away from the characteristic locus, $\gamma$ is horizontal if and only if $\dot{\tilde{\gamma}} \in$ ker $\omega_{\mathcal{S}}$, or equivalently,

$$
\left(t_{u}+2 x y_{u}-2 y x_{u}\right) \dot{u}+\left(t_{v}+2 x y_{v}-2 y x_{v}\right) \dot{v}=0
$$

where the dot denotes $d / d s$. In this case,

$$
\dot{\gamma}=\left(x_{u} \dot{u}+x_{v} \dot{v}\right) X+\left(y_{u} \dot{u}+y_{v} \dot{v}\right) Y .
$$

Proof. We only prove the first statement. We have

$$
\begin{aligned}
\gamma \text { horizontal } & \Longleftrightarrow \omega\left(\dot{\gamma}^{h}\right)=0 \\
& \Longleftrightarrow \omega\left(\sigma_{*} \dot{\tilde{\gamma}}\right)=0 \\
& \Longleftrightarrow\left(\sigma^{*} \omega\right)(\dot{\tilde{\gamma}})=0 \\
& \Longleftrightarrow \dot{\tilde{\gamma}} \in \operatorname{ker} \omega_{\mathcal{S}} .
\end{aligned}
$$

The proof of the following proposition is in [17]. For clarity, we also sketch it here. 
Proposition 3.8. The 1-form $\omega_{\mathcal{S}}$ defines an integrable foliation of $\mathcal{S}$ (with singularities at characteristic points) by horizontal surface curves. These curves are tangent to $\mathbb{J} \nu_{\mathcal{S}}^{h}$.

Proof. Integrability is immediate: $\omega_{\mathcal{S}}$ is a 1-form defined in a two-dimensional manifold. We set now

$$
\alpha=\frac{1}{\left\|N_{\sigma}^{h}\right\|}\left(t_{u}-2 y x_{u}+2 x y_{u}\right), \quad \beta=\frac{1}{\left\|N_{\sigma}^{h}\right\|}\left(t_{v}-2 y x_{v}+2 x y_{v}\right)
$$

where $\left\|N_{\sigma}^{h}\right\|=\left\|\left(\sigma_{u} \wedge^{\mathfrak{H}} \sigma_{v}\right)^{h}\right\|$. If

$$
J \mathcal{V}=\beta \frac{\partial}{\partial u}-\alpha \frac{\partial}{\partial v} \in \operatorname{ker} \omega_{\mathcal{S}}
$$

then straightforward calculations deduce $\sigma_{*}(J \mathcal{V})=\rrbracket \nu_{\mathcal{S}}$. The integral curves of $\rrbracket \nu_{\mathcal{S}}$ are the solutions of the system of differential equations $\dot{u}=\beta$ and $\dot{v}=-\alpha$.

Definition 3.9. The foliation of $\mathcal{S}$ by the integrable curves of $\mathbb{J} \nu_{\mathcal{S}}$ is called the horizontal flow of $\mathcal{S}$.

3.3. Horizontal Area and Horizontal Area Integral. In an arbitrary regular surface $\mathcal{S}$, the notion of the area $\mathcal{A}$ is given by integrating at each coordinate neighborhood $(U, \sigma)$ the length of the normal vector $N_{\sigma}=\sigma_{u} \times \sigma_{v}$. Accordingly, we define the horizontal area (elsewhere called the perimeter) of $\mathcal{S}$.

Definition 3.10. Let $\mathcal{S}$ be a regular surface in $\mathfrak{H}$ and suppose that $\sigma: U \rightarrow \mathfrak{H}$ is any surface patch. Let $N_{\sigma}^{h}=\left(\sigma_{u} \wedge^{\mathfrak{H}} \sigma_{v}\right)^{h}$. If $R$ is a domain in $U$ then, its horizontal area is given by

$$
\mathcal{A}_{\sigma}^{h}(R)=\iint_{R}\left\|N_{\sigma}^{h}(u, v)\right\| d u d v
$$

The above integral may of course be infinite; however, assuming that $R$ is contained in a rectangle whose closure lies inside $U$, then the integral is finite. Furthermore, a reparametrisation does not change the value of the integral. Finally, in the case where $\mathcal{S}$ is compact, the horizontal area of $\mathcal{S}$ is well defined and will be denoted by

$$
\mathcal{A}^{h}(\mathcal{S})=\iint_{\mathcal{S}} d \mathcal{S}^{h}
$$

Here $d \mathcal{S}^{h}$ is the horizontal area element of $\mathcal{S}$; at each surface patch $(U, \sigma)$,

$$
d \mathcal{S}^{h}=\left\|N_{\sigma}^{h}(u, v)\right\| d u d v .
$$

With the assumptions of Definition 3.10 suppose also that $\rho: \mathcal{S} \rightarrow \mathbb{R}$ is a function. The horizontal area integral of $\rho$ in $R$ is defined by

$$
\iint_{\sigma(R)} \rho d \mathcal{S}^{h}=\iint_{U} \rho(\sigma(u, v))\left\|N_{\sigma}^{h}(u, v)\right\| d u d v
$$

if $\rho\left(\sigma(u, v)\left\|N_{\sigma}^{h}(u, v)\right\| \in L^{1}(R)\right.$. Again, a reparametrisation does not change the integral and in the case where $\mathcal{S}$ is compact the horizontal area integral of $\rho$ is defined globally as follows. Suppose that $\sigma_{i}: U_{i} \rightarrow \mathcal{S}, i \in I$ is a finite covering of $\mathcal{S}$ by surface patches and $\rho \sigma_{i}\left\|N_{\sigma_{i}}^{h}\right\| \in L^{1}\left(U_{i}\right)$ for each $i \in I$. Then

$$
\iint_{\mathcal{S}} \rho d \mathcal{S}^{h}=\sum_{i \in I} \iint_{U_{i}} \rho\left(\sigma_{i}\left(u_{i}, v_{i}\right)\right) \| N_{\sigma_{i}}^{h}\left(u_{i}, v_{i}\right) d u_{i} d v_{i}
$$


3.4. Regular Surfaces and Contact-Quasiconformal Transformations. Let $\mathcal{S}$ and $\widetilde{\mathcal{S}}$ be two regular oriented surfaces in $\mathfrak{H}$. In this work we only consider mappings $\mathcal{S} \rightarrow \widetilde{\mathcal{S}}$ that are induced by $\mathcal{C}^{2}$ orientation-preserving contact transformations $f=\left(f_{1}, f_{2}, f_{3}\right)$ of $\mathfrak{H}: f^{*} \omega=\lambda \omega$ where $\lambda=J_{f}^{1 / 2}>0$. Let $f$ be such a transformation with the property $f(\mathcal{S})=\widetilde{\mathcal{S}}$. Since $f$ is a $\mathcal{C}^{2}$ diffeomorphism and both $\mathcal{S}$ and $\widetilde{\mathcal{S}}$ are $\mathcal{C}^{2}$ embedded submanifolds of $\mathfrak{H}$, it follows that the restriction $f_{\mathcal{S}}: \mathcal{S} \rightarrow \widetilde{\mathcal{S}}$ of $f$ to $\mathcal{S}$ is also a $\mathcal{C}^{2}$ diffeomorphism between $\mathcal{S}$ and $\widetilde{\mathcal{S}}$. In particular, for every local charts $(U, \sigma)$ and $(\widetilde{U}, \tilde{\sigma})$ of $\mathcal{S}$ and $\widetilde{\mathcal{S}}$, respectively, the mapping $\tilde{\sigma}^{-1} \circ f \circ \sigma$ is a $\mathcal{C}^{2}$ diffeomorphism in its domain of definition. Being also contact, the transformation $f$ adds something more to this, i.e., the surfaces $\mathcal{S}$ and $\widetilde{\mathcal{S}}$ are locally contactomorphic.

Proposition 3.11. Let $\mathcal{S}, \widetilde{\mathcal{S}}$ be two regular oriented surfaces in $\mathfrak{H}$ and $f=\left(f_{1}, f_{2}, f_{3}\right)$ be a $\mathcal{C}^{2}$ orientation-preserving contact transformation of $\mathfrak{H}$ such $f(\mathcal{S})=\widetilde{\mathcal{S}}$. Then $\mathcal{S}$ and $\widetilde{\mathcal{S}}$ are locally contactomorphic.

Proof. If $\iota_{\mathcal{S}}$ and $\iota_{\widetilde{\mathcal{S}}}$ are the inclusions of $\mathcal{S}$ and $\widetilde{\mathcal{S}}$ respectively in $\mathfrak{H}$ then $f \circ \iota_{\mathcal{S}}=\iota_{\widetilde{\mathcal{S}}} \circ f$. The result follows.

The next lemma is useful for our subsequent discussion.

Lemma 3.12. Let $\mathcal{S}$ be a regular oriented surface in $\mathfrak{H}$ and $f=\left(f_{1}, f_{2}, f_{3}\right)$ be a $\mathcal{C}^{2}$ orientationpreserving contact transformation of $\mathfrak{H}$ such that $f^{*} \omega=\lambda \omega$ where $\lambda=J_{f}^{1 / 2}$ and $J_{f}$ is the Jacobian determinant of $f$. Then the following hold.

(1) If $(U, \sigma)$ is a surface patch of $\mathcal{S}$, then $(U, f \circ \sigma)$ is a surface patch for $\widetilde{\mathcal{S}}=f(\mathcal{S})$.

(2) If $N_{\sigma}^{h}=n_{1} X+n_{2} Y$ is the horizontal normal vector of $\sigma$, then,

$$
N_{f \circ \sigma}^{h}=\lambda\left(\left(n_{1} Y f_{2}-n_{2} X f_{2}\right) X+\left(n_{2} X f_{1}-n_{1} Y f_{1}\right) Y\right) .
$$

Proof. The proof of (1) is immediate since the restriction of $f$ in $\mathcal{S}$ is a $\mathcal{C}^{2}$ diffeomorphism. To prove $(2)$ we first write the matrices of $f_{*}$ and $\sigma_{*}$ with respect to the basis $\{X, Y, T\}$. Those are

$$
\left(\begin{array}{ccc}
X f_{1} & Y f_{1} & T f_{1} \\
X f_{2} & Y f_{2} & T f_{2} \\
0 & 0 & \lambda
\end{array}\right) \quad \text { and } \quad\left(\begin{array}{cc}
x_{u} & x_{v} \\
y_{u} & y_{v} \\
\alpha\left\|N_{\sigma}^{h}\right\| & \beta\left\|N_{\sigma}^{h}\right\|
\end{array}\right)
$$

respectively, where $\alpha$ and $\beta$ are as in 3.5. Therefore, from chain rule we have

$$
\begin{aligned}
f_{*} \sigma_{u}= & \left(x_{u} X f_{1}+y_{u} Y f_{1}+\alpha\left\|N_{\sigma}^{h}\right\| T f_{1}\right) X \\
& +\left(x_{u} X f_{2}+y_{u} Y f_{2}+\alpha\left\|N_{\sigma}^{h}\right\| T f_{2}\right) Y \\
& +\lambda \alpha\left\|N_{\sigma}^{h}\right\| T,
\end{aligned}
$$

and

$$
\begin{aligned}
f_{*} \sigma_{v}= & \left(x_{v} X f_{1}+y_{v} Y f_{1}+\beta\left\|N_{\sigma}^{h}\right\| T f_{1}\right) X \\
& +\left(x_{v} X f_{2}+y_{v} Y f_{2}+\beta\left\|N_{\sigma}^{h}\right\| T f_{2}\right) Y \\
& +\lambda \beta\left\|N_{\sigma}^{h}\right\| T .
\end{aligned}
$$

The desired equation (3.10) now follows from formula (3.1). 
Proposition 3.13. With the hypotheses of Lemma 3.12, in surface patches $(U, \sigma)$ and $(U, f \circ \sigma)$ of $\mathcal{S}$ and $f(\mathcal{S})$ respectively and at non-characteristic points, the following inequality holds.

$$
\lambda\left(\left|Z f_{I}\right|-\left|\bar{Z} f_{I}\right|\right)\left\|N_{\sigma}^{h}\right\| \leq\left\|N_{f \circ \sigma}^{h}\right\| \leq \lambda\left(\left|Z f_{I}\right|+\left|\bar{Z} f_{I}\right|\right)\left\|N_{\sigma}^{h}\right\|,
$$

where $\lambda=\left|Z f_{I}\right|^{2}-\left|\bar{Z} f_{I}\right|^{2}=J_{f}^{1 / 2}$ and $J_{f}$ is Jacobian determinant of $f$.

Proof. We engage complex terminology and we write $m=n_{1}+i n_{2}$. In this manner,

$$
\begin{aligned}
& n_{1} Y f_{2}-n_{2} X f_{2}=\Re\left(m\left(Z f_{I}-Z \bar{f}_{I}\right)\right), \\
& n_{2} X f_{1}-n_{1} Y f_{1}=\Im\left(m\left(Z f_{I}+Z \bar{f}_{I}\right)\right),
\end{aligned}
$$

and therefore, equation (3.10) may be written equivalently as

$$
N_{f \circ \sigma}^{h}=2 \lambda \Re\left(\left(m Z f_{I}-\bar{m} \bar{Z} f_{I}\right) \cdot Z\right)
$$

and subsequently,

$$
\left\|N_{f \circ \sigma}^{h}\right\|=\lambda\left|Z f_{I}-e^{-2 i \arg (m)} \bar{Z} f_{I}\right|\left\|N_{\sigma}^{h}\right\| .
$$

Inequality (3.11) follows by applying the triangle inequality.

Corollary 3.14. With the hypotheses of Proposition 3.13, suppose also that $f$ is quasiconformal with Beltrami coefficient $\mu$. Then:

(1) The right inequality in (3.11) is attained as an equality if and only if

$$
\mu e^{-2 i \arg (m)}<0, \text { equivalently } \arg \mu=\pi+2 \arg m .
$$

(2) The left inequality in (3.11) is attained as an equality if and only if

$$
\mu e^{-2 i \arg (m)}>0, \text { equivalently } \arg \mu=2 \arg m .
$$

Proof. If $f$ is quasiconformal with Beltrami coefficient $\mu$, then $\bar{Z} f_{I} / Z f_{I}=\mu$, with $\mu$ essentially bounded by a constant less than 1 . Therefore,

$$
\left\|N_{f \circ \sigma}^{h}\right\|=\lambda\left|Z f_{I}\left\|1-\mu e^{-2 i \arg (m)} \mid \cdot\right\| N_{\sigma}^{h} \|\right.
$$

and inequality (3.11) may be written as

$$
|1-| \mu|| \leq\left|1-\mu e^{-2 i \arg (m)}\right| \leq|1+| \mu|| .
$$

The proof follows.

In the case where (3.13) holds, we say that $f$ has the minimal stretching property $(m S P)$ for $\mathcal{S}$.

\section{Modulus of Surface Families, Modulus Inequality, Modulus method}

In this section we define the modulus of a family $\Sigma$ of regular surfaces in $\mathfrak{H}$ (Section 4.1). The Modulus Inequality (Theorem 4.2) is proved in Section 4.2. Finally the Modulus Method and the proof of Theorem 1.4 are in Section 4.3 . 
4.1. Modulus of Surface Families. By $\Sigma$ we shall denote a family of regular surfaces in $\mathfrak{H}$. The set $\operatorname{Adm}(\Sigma)$ comprises of non-negative Borel functions $\rho$ in $\mathfrak{H}$ such that for every $\mathcal{S} \in \Sigma$ we have

$$
\iint_{\mathcal{S}} \rho d \mathcal{S}^{h} \geq 1
$$

Definition 4.1. The modulus of a family $\Sigma$ of regular surfaces in $\mathfrak{H}$ is defined by

$$
\operatorname{Mod}(\Sigma)=\inf _{\rho \in \operatorname{Adm}(\Sigma)} \iiint_{\mathfrak{H}} \rho^{4 / 3} d \mathcal{L}^{3}
$$

where by $d \mathcal{L}^{3}$ we denote the Lebesgue measure in $\mathbb{R}^{3}$.

If the infimum is attained by a function $\rho_{0} \in \operatorname{Adm}(\Sigma)$, that is

$$
\operatorname{Mod}(\Sigma)=\iiint_{\mathfrak{H}} \rho_{0}^{4 / 3} d \mathcal{L}^{3},
$$

then we call $\rho_{0}$ an extremal density for $\Sigma$.

\subsection{The Modulus Inequality.}

Theorem 4.2. Let $\Omega$ and $\Omega^{\prime}$ be domains in $\mathfrak{H}$ and $f: \Omega \rightarrow \Omega^{\prime}$ be a $\mathcal{C}^{2}$ orientation-preserving contact quasiconformal transformation. For any family of oriented regular surfaces inside $\Omega$ we have

$$
\operatorname{Mod}(f(\Sigma)) \leq \iiint_{\Omega} K_{f}^{2 / 3}(p) \rho^{4 / 3}(p) d \mathcal{L}^{3}(p) \quad \text { for each } \quad \rho \in \operatorname{Adm}(\Sigma)
$$

If moreover $K_{f}$ is the maximal distortion of $f\left(K_{f}(p) \leq K_{f}\right.$ for all $\left.p\right)$, then

$$
\frac{1}{K_{f}^{2 / 3}} \operatorname{Mod}(\Sigma) \leq \operatorname{Mod}(f(\Sigma)) \leq K_{f}^{2 / 3} \operatorname{Mod}(\Sigma) .
$$

Proof. For every $\rho \in \operatorname{Adm}(\Sigma)$ we define a non-negative Borel function in $\Omega^{\prime}$ by the relation

$$
\rho^{\prime}=\left\{\begin{array}{cc}
\frac{\rho}{\lambda\left(\left|Z f_{I}\right|-\left|\bar{Z} f_{I}\right|\right)} \circ f^{-1} & \text { in } \Omega, \\
0 & \text { elsewhere }
\end{array}\right.
$$

where $\lambda=\left|Z f_{I}\right|^{2}-\left|\bar{Z} f_{I}\right|^{2}=J_{f}^{1 / 2}$. Then for each $\mathcal{S} \in \Sigma$ with $f(\mathcal{S})=\mathcal{S}^{\prime}$ we have by the left hand side of inequality (3.11) that

$$
\iint_{\mathcal{S}^{\prime}} \rho^{\prime} d \mathcal{S}^{\prime h} \geq \iint_{\mathcal{S}} \rho d \mathcal{S}^{h}
$$

Therefore by changing the variables $q=f(p)$ we obtain

$$
\begin{aligned}
\iiint_{\Omega^{\prime}}\left(\rho^{\prime}\right)^{4 / 3}(q) d \mathcal{L}^{3}(q) & =\iiint_{\Omega}\left(\rho^{\prime}(f(p))^{4 / 3} J_{f}(p) d \mathcal{L}^{3}(p)\right. \\
& =\iiint_{\Omega} \rho^{4 / 3}(p)\left(\frac{\left|Z f_{I}(p)\right|+\left|\bar{Z} f_{I}(p)\right|}{\left|Z f_{I}(p)\right|-\left|\bar{Z} f_{I}(p)\right|}\right)^{2 / 3} d \mathcal{L}^{3}(p) \\
& =\iiint_{\Omega} K_{f}^{2 / 3}(p) \rho^{4 / 3}(p) d \mathcal{L}^{3}(p)
\end{aligned}
$$


By taking the infimum over all functions in $\operatorname{Adm}(f(\Sigma))$ we obtain (4.1). Also the right hand side of (4.2) is obtained by (4.1) and the relation

$$
K_{f}(p) \leq K_{f} \text { for all } p \in \Omega .
$$

To obtain the left hand side of (4.2) we consider the inverse transformation $f^{-1}: \Omega^{\prime} \rightarrow \Omega$ which is also quasiconformal with maximal distortion $K_{f}$. Thus, by applying (4.1) we have

$$
\begin{aligned}
\operatorname{Mod}(\Sigma)=\operatorname{Mod}\left(f^{-1}\left(\Sigma^{\prime}\right)\right) & \leq \iiint_{\Omega^{\prime}} K_{f}^{2 / 3}(q) \rho^{4 / 3}(q) d \mathcal{L}^{3}(q) \\
& \leq K_{f}^{2 / 3} \iiint_{\Omega^{\prime}} \rho^{4 / 3}(q) d \mathcal{L}^{3}(q) \text { for all } \rho \in \operatorname{Adm}\left(\Sigma^{\prime}\right)
\end{aligned}
$$

and the inequality follows after taking the infimum over all $\rho \in \operatorname{Adm}\left(\Sigma^{\prime}\right)$.

Corollary 4.3. The modulus of surface families is a conformal invariant.

4.3. The Modulus Method-Proof of Theorem 1.4. In this section we prove Theorem 1.4. We need the following two lemmas.

Lemma 4.4. Let $\Omega$ be a domain in $\mathfrak{H}, J \subset \mathbb{R}$ and $U \subset \mathbb{R}^{2}$ be open sets. Suppose that $\Phi: U \times J \rightarrow \Omega$, $((u, v), \tau) \rightarrow \Phi(u, v, \tau)$ is a diffeomorphism which foliates $\Omega$ so that the following properties hold.

(1) For every $\tau \in J$, the surface patch $\sigma_{\tau}: U \rightarrow \Omega$ defined by $\sigma_{\tau}(u, v)=\Phi(u, v, \tau)$ is regular.

(2) The horizontal normal $N_{\sigma_{\tau}}^{h}$ of each $\sigma_{\tau}$ is not zero a.e..

(3) There is a decomposition of the Lebesgue measure

$$
d \mathcal{L}^{3}(\Phi(u, v, \tau))=\left\|N_{\sigma_{\tau}}^{h}(u, v)\right\|^{4 / 3} d u d v d \mu(\tau)
$$

where $d \mu(\tau)$ is a measure on $J$.

Then, the function

$$
\rho_{0}(p)=\left\{\begin{array}{cc}
\left(|U| \cdot\left\|N_{\sigma_{\tau}}^{h}(u, v)\right\|\right)^{-1} & p=\sigma_{\tau}(u, v), \\
0 & \text { elsewhere },
\end{array}\right.
$$

where $|U|=\operatorname{Area}(U)$, is extremal for the surface family $\Sigma_{0}=\left\{\sigma_{\tau} \mid \tau \in J\right\}$ and moreover

$$
\operatorname{Mod}\left(\Sigma_{0}\right)=|U|^{-1 / 3} \int_{J} d \mu(\tau) .
$$

Proof. The density $\rho_{0}$ is admissible for $\Sigma_{0}$ :

$$
\iint_{U} \rho_{0}\left(\sigma_{\tau}(u, v)\right)\left\|N_{\sigma_{\tau}}^{h}(u, v)\right\| d u d v=|U|^{-1} \iint_{U} d u d v=1
$$

and furthermore

$$
\begin{aligned}
\iiint_{\Omega} \rho_{0}^{4 / 3}(p) d \mathcal{L}^{3}(p) & =\int_{J}\left(\iint_{U} \rho_{0}^{4 / 3}\left(\sigma_{\tau}(u, v)\right)\left\|N_{\sigma_{\tau}}^{h}(u, v)\right\|^{4 / 3} d u d v\right) d \mu(\tau) \\
& =|U|^{-1 / 3} \int_{J} d \mu(\tau) .
\end{aligned}
$$

Thus

$$
\operatorname{Mod}\left(\Sigma_{0}\right) \leq|U|^{-1 / 3} \int_{J} d \mu(\tau)
$$


For the reverse equality, we consider an arbitrary density $\rho$ such that

$$
1 \leq \iint_{U} \rho\left\|N_{\sigma_{\tau}}^{h}(u, v)\right\| d u d v
$$

and we apply Hölder's inequality:

$$
\begin{aligned}
1 & \leq\left(\iint_{U} \rho^{4 / 3}\left\|N_{\sigma_{\tau}}^{h}(u, v)\right\|^{4 / 3} d u d v\right)^{3 / 4}\left(\iint_{U} d u d v\right)^{1 / 4} \\
\therefore \quad \iint_{U} \rho^{4 / 3}\left\|N_{\sigma_{\tau}}^{h}(u, v)\right\|^{4 / 3} d u d v & \geq|U|^{-1 / 3} \\
\therefore \quad \iiint_{\Omega} \rho^{4 / 3} d \mathcal{L}^{3} & \geq|U|^{-1 / 3} \int_{J} d \mu(\tau) .
\end{aligned}
$$

The result follows after taking the infimum over all $\rho \in \operatorname{Adm}\left(\Sigma_{0}\right)$.

Lemma 4.5. Let $f_{0}: \Omega \rightarrow \Omega^{\prime}$ be an orientation-preserving quasiconformal diffeomorphism between domains in $\mathfrak{H}$. Let $\Phi$ be a foliation of $\Omega$ and $\Sigma_{0}$ be a family of surfaces as in Lemma 4.5. Assume in addition that $f_{o}$ fas the $m S P$ for the family $\Sigma_{0}$ and that

$$
K_{f_{0}}(\sigma((u, v), \tau)) \equiv K_{f_{0}}(\tau)
$$

for all $((u, v), \tau) \in U \times J$. Then

$$
\operatorname{Mod}\left(f_{0}\left(\Sigma_{0}\right)\right)=|U|^{-1 / 3} \int_{J} K_{f_{0}}^{2 / 3}(\tau) d \mu(\tau)=\iiint_{\Omega} K_{f_{0}}^{2 / 3}(p) \rho_{0}^{4 / 3}(p) d \mathcal{L}^{3}(p) .
$$

Proof. Let $\rho^{\prime} \in \operatorname{Adm}\left(f_{0}\left(\Sigma_{0}\right)\right)$ be an arbitrary density and let $\tilde{\sigma}=f_{0} \circ \sigma$. For convenience we shall write $(\mathbf{u}, \tau)$ instead of $((u, v), \tau)$. We have

$$
\begin{aligned}
1 & \leq \iint_{U} \rho^{\prime}(\tilde{\sigma}(\mathbf{u}, \tau))\left\|N_{\tilde{\sigma}}^{h}(\mathbf{u})\right\| d u d v \\
& \left.=\iint_{U} \rho^{\prime}(\tilde{\sigma}(\mathbf{u}, \tau))\right) J_{f_{0}}^{1 / 2}(\sigma(\mathbf{u}, \tau))\left(\left|Z\left(f_{0}\right)_{I}\right|-\left|\bar{Z}\left(f_{0}\right)_{I}\right|\right)_{\sigma(\mathbf{u}, \tau)}\left\|N_{\sigma}^{h}(\mathbf{u})\right\| d u d v,
\end{aligned}
$$

since $f_{0}$ has the mSP for the family $\Sigma_{0}$. By applying Hölder's inequality we have

$$
|U|^{-1 / 3} \leq \iint_{U}\left(\rho^{\prime}(\tilde{\sigma}(\mathbf{u}, \tau))\right)^{4 / 3} J_{f_{0}}^{2 / 3}(\sigma(\mathbf{u}, \tau))\left(\left|Z\left(f_{0}\right)_{I}\right|-\left|\bar{Z}\left(f_{0}\right)_{I}\right|\right)_{\sigma(\mathbf{u}, \tau)}^{4 / 3}\left\|N_{\sigma}^{h}(\mathbf{u})\right\|^{4 / 3} d u d v .
$$

By taking advantage of the assumption that the distortion function is constant along the surfaces of $\Sigma_{0}$, we multiply both sides of the inequality by $K_{f_{0}}(\tau)^{2 / 3}$ and integrate afterwards over $J$ with respect to $\mu$. Then,

$$
\left.\mid U^{-1 / 3} \int_{J} K_{f_{0}}^{2 / 3}(\tau) d \mu(\tau) \leq \int_{J} \iint_{U}\left(\rho^{\prime}\left(f_{0} \circ \sigma\right)((u, v), \tau)\right)\right)^{4 / 3} J_{f_{0}}(\sigma(\mathbf{u}, \tau))\left\|N_{\sigma}^{h}(\mathbf{u})\right\|^{4 / 3} d u d v d \mu(\tau),
$$

and by changing the variables, this yields

$$
|U|^{-1 / 3} \int_{J} K_{f_{0}}^{2 / 3}(\tau) d \mu(\tau) \leq \iiint_{\Omega}\left(\rho^{\prime}\left(f_{0}(p)\right)\right)^{4 / 3} J_{f_{0}}(p) d \mathcal{L}^{3}(p)=\iiint_{\Omega^{\prime}}\left(\rho^{\prime}(q)\right)^{4 / 3} d \mathcal{L}^{3}(q) .
$$

Since $\rho^{\prime}$ is arbitrary, we obtain

$$
\operatorname{Mod}\left(f_{0}\left(\Sigma_{0}\right)\right) \geq|U|^{-1 / 3} \int_{J} K_{f_{0}}^{2 / 3}(\tau) d \mu(\tau)
$$


We consider now the density $\rho_{0}^{\prime}$ given by

$$
\rho^{\prime}(q)=\left\{\begin{array}{cc}
\left(|U|\left(J_{f_{0}}(\sigma(\mathbf{u}, \tau))\right)^{1 / 2}\left(\left|Z\left(f_{0}\right)_{I}\right|-\left|\bar{Z}\left(f_{0}\right)_{I}\right|\right)_{\sigma(\mathbf{u}, \tau))}\left\|N_{\sigma}^{h}(\mathbf{u})\right\|\right)^{-1} & q=f(p), p=\sigma(\mathbf{u}, \tau), \\
0 & \text { elsewhere }
\end{array}\right.
$$

It is admissible for $f_{0}\left(\Sigma_{0}\right)$ :

$$
\iint_{U} \rho_{0}^{\prime}(\tilde{\sigma}(\mathbf{u}, \tau))\left\|N_{\tilde{\sigma}}^{h}(\mathbf{u})\right\| d u d v=|U|^{-1} \iint_{U} d u d v=1 .
$$

Hence,

$$
\begin{aligned}
\operatorname{Mod}\left(f_{0}\left(\Sigma_{0}\right)\right) & \leq \iiint_{\Omega^{\prime}}\left(\rho_{0}^{\prime}(q)\right)^{4 / 3} d \mathcal{L}^{3}(q)=\iiint_{\Omega}\left(\rho_{0}^{\prime}\left(f_{0}(p)\right)\right)^{4 / 3} J_{f_{0}}(p) d \mathcal{L}^{3}(p) \\
& =\int_{I} \iint_{U}\left(\rho_{0}^{\prime}(\tilde{\sigma}(\mathbf{u}, \tau))\right)^{4 / 3} J_{f_{0}}(\tilde{\sigma}(\mathbf{u}, \tau))\left\|N_{\sigma}^{h}(\mathbf{u})\right\|^{4 / 3} d u d v d \mu(\tau) \\
& =\int_{I} \iint_{U}|U|^{-4 / 3} K_{f_{0}}^{2 / 3}(\tau) d u d v d \mu(\tau)=|U|^{1 / 3} \int_{J} K_{f_{0}}^{2 / 3}(\tau) d \mu(\tau) .
\end{aligned}
$$

We show finally that

$$
\operatorname{Mod}\left(f_{0}\left(\Sigma_{0}\right)\right)=\iiint_{\Omega} K_{f_{0}}^{2 / 3}(p) \rho_{0}^{4 / 3}(p) d \mathcal{L}^{3}(p)
$$

by explicitly calculating the integral $I$ on the right:

$$
\begin{aligned}
I & =|U|^{-4 / 3} \iiint_{U \times J} K_{f_{0}}^{2 / 3}(\tau)\left\|N_{\sigma_{\tau}}^{h}(u, v)\right\|^{-4 / 3} \cdot\left\|N_{\sigma_{\tau}}^{h}(u, v)\right\|^{-4 / 3} d u d v d \mu(\tau) \\
& =|U|^{-1 / 3} \int_{J} K_{f_{0}}^{2 / 3}(\tau) d \mu(\tau) \\
& =\operatorname{Mod}\left(f_{0}\left(\Sigma_{0}\right)\right) .
\end{aligned}
$$

Proof of Theorem 1.4. Since $\rho_{0}$ is admissible for the larger family $\Sigma$ we have $\operatorname{Mod}(\Sigma)=\operatorname{Mod}\left(\Sigma_{0}\right)$. From our assumption $\operatorname{Mod}\left(f_{0}\left(\Sigma_{0}\right)\right) \leq \operatorname{Mod}(f(\Sigma))$ for each $f \in \mathcal{F}$ we have

$$
\mathfrak{M}_{2 / 3}\left(f_{0}, \rho_{0}\right)=\frac{\operatorname{Mod}\left(f_{0}\left(\Sigma_{0}\right)\right)}{\operatorname{Mod}\left(\Sigma_{0}\right)} \leq \frac{\operatorname{Mod}(f(\Sigma))}{\operatorname{Mod}(\Sigma)} \leq \mathfrak{M}_{2 / 3}\left(f, \rho_{0}\right),
$$

where for the last inequality we have used the Modulus Inequality (4.1).

\section{Proof of the Main Theorem}

In this section we prove Theorem 1.3. The proof relies heavily in the use of logarithmic coordinates for $\mathfrak{H}$; an overview is in Section 5.1. We review in brief some known facts about the stretch map in Section 5.2. Next, the proof is given in three steps. In Step 1 we calculate the $\operatorname{modulus} \operatorname{Mod}\left(\Sigma_{0}\right)$ and the extremal density $\rho_{0}$ of our prototype family $\Sigma_{0}$ which consists of pieces of Heisenberg cones lying inside the spherical ring $S_{a, b}$. We do this using Lemma 4.4, the foliation here is given by the logarithmic coordinates parametrisation $\Phi$. In the second step of the proof we show that $f_{k}$ satisfies the assumptions of Lemma 4.5 and in order to apply Theorem 1.4 and conclude the proof, we show in the third step that there exists a wider surface family $\Sigma$ inside $S_{a, b}$ such that $r h o_{0} \in \operatorname{Adm}(\Sigma)$ and $f_{k}\left(\Sigma_{0}\right) \subseteq f(\Sigma)$ for each $f \in \mathcal{F}^{\prime}$. 
5.1. Logarithmic coordinates. Details about this section can be found in [18] as well as in [2]. Logarithmic coordinates for $\mathfrak{H}$ are directly analogous to logarithmic coordinates in the complex plane. These are given by the map $\Phi: \widetilde{\mathfrak{H}}_{0}=\mathbb{R} \times(-\pi / 2, \pi / 2) \times \mathbb{R} \rightarrow \mathfrak{H} \backslash \mathcal{V}=\widetilde{\mathfrak{H}}$ where $\mathcal{V}$ is the vertical axis, by the relation

$$
\Phi(\xi, \psi, \eta)=\left(i \cos ^{1 / 2} \psi e^{\frac{\xi+i(\psi-3 \eta)}{2}},-\sin \psi e^{\xi}\right) .
$$

We state in brief some known facts about logarithmic coordinates. In the following, if $f: Q \rightarrow \widetilde{\mathfrak{H}}$ is a $\mathcal{C}^{k}$ map, where $Q$ is a simply connected subset of $\widetilde{\mathfrak{H}}$ and $k \geq 0$, then $\tilde{f}$ will denote the map defined by the relation $\Phi \circ f=\tilde{f} \circ \Phi$. We shall write

$$
\tilde{f}(\xi, \psi, \eta)=(\Xi(\xi, \psi, \eta), \Psi(\xi, \psi, \eta), \mathrm{H}(\xi, \psi, \eta)) .
$$

The contact form $\omega$ has the following expression in logarithmic coordinates:

$$
\omega=-e^{\xi}(\sin \psi d \xi+3 \cos \psi d \eta)
$$

and the contact conditions (2.2), (2.3) and (2.4) for a contact $f$ are equivalent to the following conditions for $\tilde{f}$ :

$$
\begin{aligned}
& \mathrm{H}_{\psi}+\frac{1}{3} \tan \Psi \cdot \Xi_{\psi}=0, \\
& W_{\xi, \eta} \mathrm{H}+\frac{1}{3} \tan \Psi \cdot W_{\xi, \eta} \Xi=0,
\end{aligned}
$$

where $W_{\xi, \eta}=\partial_{\xi}-(\tan \psi / 3) \partial_{\eta}$. We wish to stress at this point that

$$
\Phi_{*}\left(W_{\xi, \eta}\right)=\Re\left(\frac{|z|^{2}-i t}{\bar{z}} Z\right), \quad \text { and } \quad \Phi_{*}\left(\partial_{\psi}\right)=\Im\left(\frac{|z|^{2}-i t}{\bar{z}} Z\right),
$$

generate the horizontal tangent space at each point of $\widetilde{\mathfrak{H}}$.

Finally, the Beltrami coefficient for $f$ is

$$
\mu_{f}(\Phi(\xi, \psi, \eta))=-e^{3 i(\psi-\eta)} \frac{\bar{W}(\Xi+i \Psi)}{W(\Xi+i \Psi)},
$$

where $W=W_{\xi, \eta}-i \partial_{\psi}$ and $\bar{W}=W_{\xi, \eta}+i \partial_{\psi}$.

5.2. The stretch map. The stretch map $f_{k} \mathfrak{H} \rightarrow \mathfrak{H}, k \in \mathbb{R}, k \neq 0,1$, is defined in logarithmic coordinates by

$$
\tilde{f}_{k}(\xi, \psi, \eta)=\left(k \xi, \tan ^{-1}\left(\frac{\tan \psi}{k}\right), \eta\right) .
$$

In [2] it is shown that $\tilde{f}_{k}$ satisfies the contact conditions (5.2) and (5.3) and that the Beltrami coefficient of $f_{k}$ is

$$
\mu_{f_{k}}(\Phi(\xi, \psi, \eta))=-e^{3 i(\psi-\eta)} \frac{k^{2}-1}{k^{2}+1+\tan ^{2} \psi}
$$

Since $\left\|\mu_{f_{k}}\right\|_{\infty}<1, f_{k}$ is quasiconformal in $\mathfrak{H}$. In the following, the domain of $f_{k}$ will be the spherical $\operatorname{ring} S_{a, b}=\left\{(z, t) \in \mathfrak{H}: a^{4}<|z|^{4}+t^{2}<b^{4}\right\}$ and we shall consider the parametrisation of $S_{a, b} \backslash \mathcal{V}$ by logarithmic coordinates

$$
(\xi, \psi, \eta) \mapsto \Phi(\xi, \psi, \eta)
$$


where $(\xi, \psi, \eta) \in(2 \log a, 2 \log b) \times(-\pi / 2, \pi / 2) \times(-2 \pi / 3,2 \pi / 3)$. Since the Jacobian determinant of $\Phi$ equals to $J_{\Phi}=-(3 / 4) e^{2 \xi}$, the Lebesgue mesure in $S_{a, b}$ is

$$
d \mathcal{L}^{3}(\xi, \psi, \eta)=\frac{3}{4} e^{2 \xi} d \xi d \psi d \eta
$$

We are now set for the proof of our main theorem.

Proof of Theorem 1.3. The proof shall be given in steps.

Step 1: Modulus of $\Sigma_{0}$. Our prototype family will be the foliation $\Sigma_{0}$ of the spherical ring $S_{a, b}$ by the pieces of Heisenberg cones $\mathcal{C}_{\alpha}$ lying inside $S_{a, b}$; these have cartesian equations

$$
t=-\alpha\left(x^{2}+y^{2}\right), \quad \alpha \in \mathbb{R}, \quad a^{2}<x^{2}+y^{2}<b^{2} .
$$

In logarithmic coordinates those equations are $\tan \psi=\alpha$ where $\psi \in(-\pi / 2, \pi / 2)$ and thus a cone $\mathcal{C}_{\alpha}$ may be parametrised by the single patch

$$
\sigma_{\psi}(\xi, \eta)=\Phi(\xi, \psi, \eta), \quad(\xi, \eta) \in U=(2 \log a, 2 \log b) \times(-2 \pi / 3,2 \pi / 3) .
$$

At each leaf $\mathcal{C}_{\alpha}$ of the foliation $\Sigma_{0}$, the chart $\left(U, \sigma_{\psi}\right)$ is a $\mathcal{C}^{2}$ chart in the atlas of the leaf. For the horizontal normal $N_{\sigma_{\psi}}^{h}$ we calculate

$$
\begin{aligned}
& \frac{\partial(x, y)}{\partial(\xi, \eta)}=-\frac{3}{4} e^{\xi} \cos \psi, \\
& \frac{\partial(y, t)}{\partial(\xi, \eta)}=-\frac{3}{2} e^{3 \xi / 2} \cos ^{1 / 2} \psi \sin \psi \sin ((3 \eta-\psi) / 2), \\
& \frac{\partial(t, x)}{\partial(\xi, \eta)}=-\frac{3}{2} e^{3 \xi / 2} \cos ^{1 / 2} \psi \sin \psi \cos ((3 \eta-\psi) / 2) .
\end{aligned}
$$

Therefore

$$
\begin{aligned}
& N_{\sigma_{\psi}}^{h}(\xi, \eta)=-\frac{3}{2} e^{3 \xi / 2} \cos ^{1 / 2} \psi(\cos (3(\eta-\psi) / 2) X-\sin (3(\eta-\psi) / 2) Y), \\
& \left\|N_{\sigma_{\psi}}^{h}(\xi, \eta)\right\|=\frac{3}{2} e^{3 \xi / 2} \cos ^{1 / 2} \psi .
\end{aligned}
$$

We can now use Lemma 4.4 to calculate the extremal density and the modulus of $\Sigma_{0}$.

Proposition 5.1. Let $\Sigma_{0}$ be the family of pieces of Heisenberg cones $\mathcal{C}_{\alpha}$ inside the spherical ring $S_{a, b}$. Then

$$
\operatorname{Mod}\left(\Sigma_{0}\right)=\left(2^{5} \pi \log (b / a)\right)^{-1 / 3} \mathrm{~B}(1 / 2,1 / 6),
$$

where $\mathrm{B}$ denotes the beta function. The extremal density $\rho_{0}$ is given by

$$
\rho_{0}(z, t)=\frac{1}{4 \pi \log (b / a)} \cdot \frac{1}{\left.|z||| z\right|^{2}-i t \mid} \mathcal{X}\left(S_{a, b}\right) .
$$

Proof. We apply Lemma 4.4, Here:

(1) $U=(2 \log a, 2 \log b) \times(-2 \pi / 3,2 \pi / 3), J=(-\pi / 2, \pi / 2), \Phi((\xi, \eta), \psi)=\Phi(\xi, \psi, \eta)$ and

(2) $\Sigma_{0}$ is the family $\sigma_{\psi}(\xi, \eta)=\Phi((\xi, \eta), \psi)$. 
From (5.5) we have $\left\|N_{\sigma_{\psi}}^{h}(\xi, \eta)\right\|=\frac{3}{2} e^{3 \xi / 2} \cos ^{1 / 2} \psi$ and also

$$
\left.d \mathcal{L}^{3}(\Phi((\xi, \eta), \psi))\right)=\left\|N_{\sigma_{\psi}}^{h}(\xi, \eta)\right\|^{4 / 3} d \xi d \eta d \mu(\psi),
$$

where

$$
d \mu(\psi)=\frac{1}{2}\left(\frac{3}{2}\right)^{-1 / 3} \cos ^{-2 / 3} \psi d \psi, \quad \psi \in(-\pi / 2, \pi / 2) .
$$

Formulae (5.6) and (5.7) follow immediately after short calculations.

Step 2: Modulus of $f_{k}\left(\Sigma_{0}\right)$. The stretch map has the mSP for the family of cones $\Sigma_{0}$. Indeed, from equation (5.4) we have

$$
m=m_{\sigma_{\psi}}=-\frac{3}{2} e^{3 \xi / 2} \cos ^{1 / 2} \psi e^{3 i(\psi-\eta) / 2}, \quad \arg (m)=\pi+\frac{3}{2}(\psi-\eta) .
$$

Therefore

$$
\mu_{f_{k}} e^{-2 i \arg (m)}=\frac{1-k^{2}}{k^{2}+1+\tan ^{2} \psi}
$$

which is positive if $k \in(0,1)$. Thus the distortion function $K_{f_{k}}$ is

$$
K_{f_{k}}(\Phi(\xi, \psi, \eta))=\frac{1+\tan ^{2} \psi}{k^{2}+\tan ^{2} \psi}
$$

and it is constant in each leaf of $\Sigma_{0}$ since it only depends on $\psi$. By Lemma 4.5 we have

$$
\begin{aligned}
\operatorname{Mod}\left(f_{k}\left(\Sigma_{0}\right)\right) & =\left(\frac{8 \pi}{3} \log (b / a)\right)^{-1 / 3} \int_{-\pi / 2}^{\pi / 2}\left(\frac{1+\tan ^{2} \psi}{k^{2}+\tan ^{2} \psi}\right)^{2 / 3} \cdot \frac{1}{2}\left(\frac{3}{2}\right)^{-1 / 3} \cos ^{-2 / 3} \psi d \psi \\
& =\left(2^{5} \pi \log (b / a)\right)^{-1 / 3} \int_{-\pi / 2}^{\pi / 2} \frac{1+\tan ^{2} \psi}{\left(k^{2}+\tan ^{2} \psi\right)^{2 / 3}} d \psi \\
(z=\tan \psi) & =\left(2^{5} \pi \log (b / a)\right)^{-1 / 3} \int_{-\infty}^{+\infty} \frac{d z}{\left(k^{2}+z^{2}\right)^{2 / 3}} \\
(u=z / k) & =k^{-1 / 3} \cdot\left(2^{5} \pi \log (b / a)\right)^{-1 / 3} \int_{-\infty}^{+\infty} \frac{d u}{\left(1+u^{2}\right)^{2 / 3}} \\
(v=\arctan u) & =k^{-1 / 3} \cdot\left(2^{5} \pi \log (b / a)\right)^{-1 / 3} \int_{-\pi / 2}^{\pi / 2} \cos ^{-2 / 3} v d v \\
& =k^{-1 / 3} \cdot \operatorname{Mod}\left(\Sigma_{0}\right) .
\end{aligned}
$$

Note that this equality could have been immediately deduced by the fact that $f_{k}$ maps Heisenberg cones to Heisenberg cones and the formula for the modulus of the family of Heisenberg cones inside a spherical ring. On the other hand, if $\rho_{0}$ is given by (5.7), then again by Lemma 4.5 we have

$$
\iiint_{S_{a, b}} K_{f_{k}}^{2 / 3}(p) \rho_{0}^{4 / 3}(p) d \mathcal{L}^{3}(p)=\operatorname{Mod}\left(f_{k}\left(\Sigma_{0}\right)\right) .
$$

Step 3: Conclusion of Proof. To conclude the proof of Theorem 1.3 we must define the larger family $\Sigma$ and show that $f_{k}\left(\Sigma_{0}\right) \subseteq f(\Sigma)$ and that $\rho_{0}$ is admissible for $\Sigma$. A natural choice is the one of the family of regular surfaces that join the two boundaries of the spherical ring. But this is a rather large family and it contains surfaces which might not at all look like Heisenberg cones, even locally. To that end, we examine the particular properties of Heisenberg cones. Let $\mathcal{C}$ be such a cone inside $S_{a, b}$ and let $\widetilde{\mathcal{C}}$ be $\Phi^{-1}(\mathcal{C})$. Then the following hold. 
(1) The surface $\widetilde{\mathcal{C}}$ admits a parametrisation

$$
\begin{aligned}
& \tilde{\sigma}(\xi, \eta)=(\Xi(\xi, \eta), \Psi(\xi, \eta), \mathrm{H}(\xi, \eta))=(\xi, c, \eta), \quad(\xi, \eta) \in(2 \log a, 2 \log b) \times(-2 \pi / 3,2 \pi / 3), \\
& \text { that is, } \psi=\psi(\xi, \eta)=c .
\end{aligned}
$$

(2) The induced 1 -form $\omega_{\mathcal{C}}$ is given in logarithmic coordinates by

$$
\omega_{\widetilde{\mathcal{C}}}=\Phi^{*}\left(\omega_{\mathcal{C}}\right)=-e^{\xi} \cos c(\tan c d \xi+3 d \eta)
$$

and therefore ker $\omega_{\mathcal{C}}$ is generated by $\partial_{\xi}-(\tan c / 3) \partial_{\eta}$ which may be identified to the restriction of $\Phi_{*}\left(W_{\Xi, \mathrm{H}}\right)$ on $\mathcal{C}: \widetilde{\sigma}_{*}\left(\partial_{\xi}-(\tan c / 3) \partial_{\eta}\right)=W_{\Xi, \mathrm{H}}$. Note that this also implies that the restriction of the vector field $\Phi_{*}(\partial / \partial \Psi)$ on $\mathcal{C}$ is horizontally transverse to the horizontal space of $\mathcal{C}$.

One may now check that a surface $\mathcal{S}$ which is such that $\widetilde{\mathcal{S}}=\Phi^{-1}(\mathcal{S})$ admits a local parametrisation

$$
\widetilde{\sigma}(\xi, \eta)=(\Xi(\xi, \eta), \Psi(\xi, \eta), \mathrm{H}(\xi, \eta)), \quad(\xi, \eta) \in U=(2 \log a, 2 \log b) \times(-2 \pi / 3,2 \pi / 3),
$$

has the property that ker $\omega_{\mathcal{S}}$ is generated by a vector field which can be identified to $\Phi_{*}(V)$ where

$$
V=\frac{\partial(\Xi, \mathrm{H})}{\partial(\xi, \eta)} W_{\Xi, \mathrm{H}}+3\left(\frac{\partial(\Psi, \mathrm{H})}{\partial(\xi, \eta)}-\frac{\tan \Psi}{3} \frac{\partial(\Xi, \Psi)}{\partial(\xi, \eta)} \frac{\partial}{\partial \Psi}\right) .
$$

Note that $\partial(\Xi, \mathrm{H}) / \partial(\xi, \eta) \neq 0$ allow us to reparametrise so that $\widetilde{C}$ is locally of the form $\Psi=\Psi(\xi, \eta)$.

The previous observations drive us to impose the following additional condition (C) for $\Sigma$ :

(C) Every $\mathcal{S} \in \Sigma$ is such that $\widetilde{\mathcal{S}}$ can be locally parametrised away from its characteristic locus as

$$
\Psi=\Psi(\xi, \eta), \quad(\xi, \eta) \in U=(2 \log a, 2 \log b) \times(-2 \pi / 3,2 \pi / 3) .
$$

In the first place, if $\Sigma$ satisfies $(\mathrm{C})$ then for any arbitrary contact quasiconformal map $f \in \mathcal{F}$ we have $f_{k}\left(\Sigma_{0}\right) \subseteq f(\Sigma)$. To see this, suppose that we have a Heisenberg cone $\mathcal{C} \in f_{k}\left(\Sigma_{0}\right)$ where $\widetilde{\mathcal{C}}$ is given by $\psi=c$ and an arbitrary contact qc map $f \in \mathcal{F}$. The surface $\mathcal{C}^{\prime}=f^{-1}(\mathcal{C})$ admits a local parametrisation

$$
(\xi, \eta) \mapsto(\Xi(\xi, c, \eta), \Psi(\xi, c, \eta), \mathrm{H}(\xi, c, \eta))
$$

where $(\Xi, \Psi, \mathrm{H})=\tilde{f}^{-1}$ and thus its horizontal tangent space is generated by $V$ as in (5.8). Suppose that $\partial(\Xi, \mathrm{H})=0$ in the neighborhood of some point. From the contact conditions for $f^{-1}$ we find

$$
\tan \Psi \Xi_{\xi}+3 \mathrm{H}_{\xi}=\frac{\tan c}{3}\left(\tan \Psi \Xi_{\eta}+3 \mathrm{H}_{\eta}\right)
$$

hence

$$
\frac{\tan \Psi}{3}=\frac{\tan c \mathrm{H}_{\eta}-3 \mathrm{H}_{\xi}}{3 \Xi_{\xi}-\tan c \Xi_{\eta}}
$$

The denominator is different from zero; else, we would have a quasilinear PDE $3 \Xi_{\xi}-\tan c \Xi_{\eta}=0$ whose solution is $\Xi=$ const. From the contact condition it also follows that $H=$ const. and we cannot have a surface.

Now we calculate:

$$
\begin{aligned}
\frac{\partial(\Psi, \mathrm{H})}{\partial(\xi, \eta)}-\frac{\tan \Psi}{3} \frac{\partial(\Xi, \Psi)}{\partial(\xi, \eta)} & =\Psi_{\xi} \mathrm{H}_{\eta}-\Psi_{\eta} \mathrm{H}_{\xi}-\frac{\tan \Psi}{3} \cdot\left(\Xi_{\xi} \Psi_{\eta}-\Psi_{\xi} \Xi_{\eta}\right) \\
& =\frac{\left(\Psi_{\xi} \mathrm{H}_{\eta}-\Psi_{\eta} \mathrm{H}_{\xi}\right)\left(3 \Xi_{\xi}-\tan c \Xi_{\eta}\right)-\left(\Xi_{\xi} \Psi_{\eta}-\Psi_{\xi} \Xi_{\eta}\right)\left(\tan c \mathrm{H}_{\eta}-3 \mathrm{H}_{\xi}\right)}{3 \Xi_{\xi}-\tan c \Xi_{\eta}} \\
& =\frac{3 \Psi_{\xi}-\tan c \Psi_{\eta}}{3 \Xi_{\xi}-\tan c \Xi_{\eta}} \cdot \frac{\partial(\Xi, \mathrm{H})}{\partial(\xi, \eta)}=0 .
\end{aligned}
$$


Thus $V=0$ which is a contradiction since we are away from the characteristic locus. Therefore $\mathcal{C}^{\prime} \in \Sigma$ which implies that $\mathcal{C}=f\left(\mathcal{C}^{\prime}\right) \in f(\Sigma)$.

We finally show that $\rho_{0} \in \operatorname{Adm}(\Sigma)$. For this, let $\mathcal{S} \in \Sigma$ and we may suppose that it is parametrised by the single surface patch

$$
\sigma(\xi, \eta)=(z(\xi, \eta), t(\xi, \eta))=\left(i \cos ^{1 / 2}(\psi(\xi, \eta)) e^{\frac{\xi+i(\psi(\xi, \eta)-3 \eta)}{2}},-e^{\xi} \sin \psi(\xi, \eta)\right) .
$$

This assumption is allowed by our condition (C) and the fact that the horizontal area integral does not depend on the choice of coordinates, see Section 3.3. We have

$$
\begin{aligned}
z_{\xi} & \left.=\frac{z}{2} \cdot\left(\left(1-\tan \psi \cdot \psi_{\xi}\right)+i \psi_{\xi}\right), \quad z_{\eta}=\frac{z}{2} \cdot\left(-\tan \psi \cdot \psi_{\eta}\right)+i\left(\psi_{\eta}-3\right)\right), \\
t_{\xi} & =-e^{\xi}\left(\sin \psi+\cos \psi \cdot \psi_{\xi}\right), \quad t_{\eta}=-e^{\xi} \cos \psi \cdot \psi_{\eta} .
\end{aligned}
$$

Therefore

$$
\begin{gathered}
\sigma_{\xi}=z_{\xi} Z+\overline{z_{\xi}} \bar{Z}-a T, \\
\sigma_{\eta}=z_{\eta} Z+\overline{z_{\eta}} \bar{Z}-b T,
\end{gathered}
$$

where $a=e^{\xi} \sin \psi, b=3 e^{\xi} \cos \psi$. Thus

$$
N_{\sigma}^{h}=\left(\sigma_{\xi} \wedge^{\mathfrak{H}} \sigma_{\eta}\right)^{h}=i\left(\left(b z_{\xi}-a z_{\eta}\right) Z-\left(b \overline{z_{\xi}}-a \overline{z_{\eta}}\right) \bar{Z}\right) .
$$

Straightforward calculations now yield

$$
\begin{aligned}
\left\|N_{\sigma}^{h}\right\| & =\left|b z_{\xi}-a z_{\eta}\right| \\
& =\frac{3}{2} e^{3 \xi / 2} \cos ^{1 / 2} \psi\left(1+\left(\psi_{\xi}-\frac{\tan \psi \cdot \psi_{\eta}}{3}\right)^{2}\right)^{1 / 2} .
\end{aligned}
$$

Hence

$$
\begin{aligned}
\iint_{\mathcal{S}} \rho_{0} d \mathcal{S}^{h}= & \iint_{U} \rho_{0}\left\|N_{\sigma}^{h}(\xi, \eta)\right\| d \xi d \eta \\
= & \frac{1}{4 \pi \log (b / a)} \iint_{U} e^{-3 \xi / 2} \cos ^{-1 / 2} \psi \cdot \frac{3}{2} e^{3 \xi / 2} \cos ^{1 / 2} \psi \times \\
& \times\left(1+\left(\psi_{\xi}-\frac{\tan \psi \cdot \psi_{\eta}}{3}\right)^{2}\right)^{1 / 2} d \xi d \eta \\
= & \frac{3}{8 \pi \log (b / a)} \int_{2 \log a}^{2 \log b} \int_{-2 \pi / 3}^{2 \pi / 3}\left(1+\left(\psi_{\xi}-\frac{\tan \psi \cdot \psi_{\eta}}{3}\right)^{2}\right)^{1 / 2} d \xi d \eta \\
\geq & \frac{3}{8 \pi \log (b / a)} \int_{2 \log a}^{2 \log b} \int_{-2 \pi / 3}^{2 \pi / 3} d \xi d \eta \\
= & 1 .
\end{aligned}
$$

The proof of Theorem 1.3 concludes here.

\section{REFERENCES}

[1] K. Astala \& T. Iwaniec \& G.J. Martin \& J. Onninen; Extremal mappings of finite distortion. Proc. Lon. Math. Soc. (3), (2005), 655-702.

[2] Z.M. Balogh \& K. Fässler \& I.D. Platis; Modulus method and radial stretch map in the Heisenberg group. Ann. Acad. Sci. Fenn. (38), 2013, 1-32. 
[3] Z.M Balogh \& K. Fässler \& I.D. Platis; Modulus of curve families and extremality of spiral-stretch maps. J. Anal. Math. (113), (2011), 265-291.

[4] L. Capogna \& D. Danielli \& S.D. Pauls \& J.T. Tyson; An introduction to the Heisenberg group and the sub-Riemannian isoperimetric problem. Prog. in Math. (259). Birkhuser Verlag, Basel, 2007.

[5] N.S. Dairbekov; On mappings of bounded distortion on the Heisenberg group. Sib. Math. Zh. (41), Issue 1, (2000), 49-59.

[6] M.P. Do Carmo; Riemannian geometry. Birkhäuser, XVI ed. (1992).

[7] F. Gardiner \& N. Lakic; Quasiconformal Teichmüller Theory. Math. Surv. and Mon. (76), AMS, (2000).

[8] H. Grötzsch; Über möglichst konforme Abbildungen von schlichten Bereichen. Ber. Math.-phys. Kl. Sachs. Akad. Wis. Leipsig, (84), (1932), 114-120.

[9] V. Gutlyanskii \& O. Martio; Rotation estimates and spirals. Conf. Geom. Dyn. (5), (2001), 6-20.

[10] J. Heinonen; Calculus on Carnot groups. In: Fall school in Analysis (Jyväskylä, 1994), Report 68, Univ. Jyväskylä, Jyväskylä, (1995), 1-31.

[11] B. Kirchheim \& F. Serra Cassano; Rectifiability and parametrization of intrinsic regular surfaces in the Heisenberg group. Ann. Scuola Norm. Sup. Pisa CI. Sci (5), (2004), 871-896.

[12] A. Korányi \& H.M. Reimann; Foundations for the theory of quasiconformal mappings of the Heisenberg group. Adv. in Math. (111) (1995), 1-87.

[13] A. Korányi, H.M. Reimann; Quasiconformal mappings on the Heisenberg groups. Invent. Math. (80) (1985), no.2, 309-338.

[14] G.J. Martin; The Teichmüller problem for mean distortion. Ann. Acad. Sci. Fenn. Math. (34), (2009), $233-247$.

[15] G.D. Mostow; Strong rigidity in locally symmetric spaces. Ann. Math. Stud. (78), Princeton Univ. Press, Princeton, N.J., 1973.

[16] P. Pansu; Métriques de Carnot-Carathéodory et quasiisométries des espaces symétriques de rang un. Ann. of Math. (129), (1989), 1-60.

[17] I.D. Platis; Straight ruled surfaces in the Heisenberg group. Arxiv 1212.5834v2 [math.DG]

[18] I.D. Platis; The geometry of complex hyperbolic packs. Math. Proc. Cam. Phil. (147), (2009), $205-234$.

[19] K. Strebel; Extremal quasiconformal mappings. Res. Math. (10), 1-2, (1986), 168-210.

[20] K. Strebel; Quadratic differentials. Springer-Verlag, Berlin \& New York, 1984.

[21] O. Teichmüller; Extremale quasikonforme Abbildungen und quadratische Differentiale. Abh. Preuss. Akad. Wiss. Math.-Nat. Kl. (22), (1939), 197.

[22] A. Vasil'ev; Moduli of families of curves for conformal and quasiconformal mappings. Springer-Verlag, Berlin \& New York, 2004.

[23] S.K. Vodop'yanov; Monotone functions and quasiconformal mappings on Carnot groups. Sib. Math. Zh. (37), Issue 6, (1996), 1269-1295.

Department of Mathematics and Applied Mathematics, University of Crete, University Campus, GR 70013 Heraklion Crete, Greece.

E-mail address: jplatis@math.uoc.gr 